\title{
Reduced-stress method for efficient computation of time-dependent viscoelastic flow with stress equations of FENE-P type
}

\author{
P.J. Oliveira \\ Departamento de Engenharia Electromecânica, C-MAST, Universidade da Beira Interior, 6201-001 Covilhã, Portugal
}

\section{A R T I C L E I N F O}

\section{Article history:}

Received 30 May 2017

Revised 15 August 2017

Accepted 4 September 2017

Available online 6 September 2017

\section{Keywords:}

Time-dependent viscoelastic flows

Numerical method

Stress equations

Fene-P type

Rotating duct

Coriolis effects

Computational rheology

\begin{abstract}
A B S T R A C T
Most calculation procedures for time-dependent viscoelastic flows require iteration within the time step used to advance the solution, in order to satisfy simultaneously the momentum and the constitutive equations for each stress component. We have devised a way of reformulating the constitutive equation for the FENE-P model, or models described by similar equations expressed in terms of the stress tensor, which enables iterative methods for simulating time-dependent viscoelastic flows to become much more efficient: the number of iterations to obtain a solution with the reformulated stress equations is substantially smaller (by a factor of 5-10) than with a comparable method applied to the original, nonreformulated, constitutive equations. The proposed reformulation is rather simple and consists in considering as dependent variables the reduced stresses obtained by dividing the stress components by the extensibility function of the model. It is tested with three problems of increasing complexity, start-up of channel and square-duct flows, and start-up of a rotating duct flow.
\end{abstract}

(c) 2017 Elsevier B.V. All rights reserved.

\section{Introduction}

In this work, we deal with the issue of devising more efficient numerical methods for the simulation of time-dependent viscoelastic flows. Such simulations invariably involve iterations inside each time step to tackle the explicit or implicit nonlinearities introduced by the need to solve the constitutive equations in addition to the equations of motion.

We have found that a simple modification to the non-linear, differential constitutive equations of the FENE-P type, in which the stress tensor is the dependent variable, results in a considerable reduction of the total number of iterations required for a given simulation, thus representing a gain of efficiency which may amount to one order of magnitude. In addition to the original FENE-P [1,2], another model which also falls into that type and may benefit from the method here devised is the constant-viscosity FENE-CR model [3]. However, we want to emphasize from the outset that quasilinear rheological models, such as the upper-convected Maxwell or the Oldroyd-B [4] models, cannot benefit from the proposed approach, as cannot the non-conservative models (in the sense used by Oliveira [5]), such as the Phan-Thien/Tanner (PTT) [6] or the FENE-MCR [7] - in fact, these are the models which are often associated with "easier" numerical simulations eg. [8]). In a way, it may be resumed that the present method transforms a FENE-P equa-

E-mail address: pjpo@ubi.pt tion into a PTT-type equation, thus benefiting from the smoother numerical characteristics associated with the latter.

In our simulations, we employ a finite volume method (FVM) [9] but the proposed approach is not at all connected to a specific methodology to solve the equations and therefore it can easily be applied to finite element methods or other numerical methodologies.

The emphasis on directing research efforts toward the simulation of time-dependent non-Newtonian flows has been recognised for some time: in 1992, at the VIIth Workshop on Numerical Methods in Non-Newtonian Flow it is stated "The development of timedependent simulations was identified as a major issue for the future" [10]; in 1993, at the VIIIth Workshop, "transient benchmarks" were defined [11]; and in 1995, the IXth Workshop "saw the start of significant effort on 3D, time-dependent flows when viscoelasticity is important" [12]. Since then many papers have appeared on such topic, which are too numerous to be reviewed here, even in a cursory way. The book by Owens and Phillip [13] gives a comprehensive view of works of different methodologies (FVM- Finite Volume Method; FEM- Finite Element Method; spectral methods) up to the year of 2002; on the FVM side, which is more pertinent to us, the paper by Xue et al. [14] is perhaps the most relevant as it provides an analysis of the various approaches available. Walters and Webster in 2003 state "seemingly two-dimensional steady problems often need to be treated as three-dimensional and unsteady" [15], once more reinforcing the need for efficient viscoelastic solvers. A review of the literature basically shows that meth- 
ods for time-dependent viscoelastic flow are imported from Newtonian CFD, with the constitutive equations appended to the calculation cycle (e.g. [16,17] with FEM or mixed FEM/FVM, [14,18] with FVM and $[19,20]$ with spectral methods rooted on FEM). A few approaches having some similarity with that proposed here, in terms of implying a reformulation of the constitutive or momentum equations with view to improve the numerical behaviour of the solution algorithm, were the EEME [21], EVSS [22] and AVSS [23] formulations, the three applied in conjunction with FEM, the grid-bygrid method [24] with FVM, all these for steady-state flows, and, more recently, the important breakthrough introduced by Fattal and Kupferman [25,26] with their log-conformation formalism. It is however fair to say that the main aspects of those numerical methodologies were not related specifically with time-dependent simulations and even the log-conformation has been essentially applied to steady-state solutions, even if the example given in the original reference [26] was a (fictitious) time-dependent flow. More recently, applications to time varying flows are arising, see e.g. [27] where the log-conformation formalism is employed to solve free-surface 3D viscoelastic flows.

In addition to the above cited works, it is relevant to mention in this short review some more recent (after 2010) formulations with FEM, especially designed for time-dependent viscoelastic flows, such as those of D'Avino, Hulsen and co-workers [28,29] who proposed and tested some variants of a decoupled semi-implicit scheme for inertialess and inertial flows, and Castillo and Codina [30] who extended the fractional-step method of classical CFD into several algorithmic arrangements for the Oldroyd-B equation, exhibiting both 2 nd and 3rd order accuracy in time. The temporal discretization of these methods was essentially based on the backward-difference formula (BDF; in $[28,29]$ it is named Gear method), or the three-time level of the present paper, except for some details. In $[28,29]$ the constitutive equations for the Oldroyd$\mathrm{B}$ and Giesekus models are solved in terms of the conformation tensor (the log-conformation was not attempted due to the increased complexity), while in [30] the Oldroyd-B equation was solved for the basic stress tensor, as in the method here suggested. An interesting point of the formulation of $[28,29]$ is that the stress in the divergence term of the momentum equation is substituted by an approximation obtained from an explicit Euler discretization of the constitutive equation, and the terms containing either the velocity gradients or the velocity itself (the convective term in the constitutive equation) are treated implicitly when solving the momentum equation for the velocity. In this way, the system representing the algebraic momentum equations is never singular, even for constitutive models without a solvent viscosity in inertialess flows, and the implicitness of the equation is augmented. It would appear that in the context of FVM such approach could be implemented at expense of a wider computational stencil (typically, FVM solvers are designed for a compact stencil containing just the closest neighbours to a given cell, that is 4 in $2 \mathrm{D}$ and 6 in 3D, for structured meshes).

To test the reduced stress method, we use time-dependent flow problems of increasing complexity, all related to the transient establishment of a viscoelastic flow in conduits. The easiest problem is the start-up of fully developed viscoleastic flow in a planar channel, which is one-dimensional (1D) in space but, in spite of its apparent simplicity, there is no available analytical solution for the FENE-P or FENE-CR models. However, simple 1D programs we have utilised in previous work [31] to simulate the start-up of OldroydB fluid, for which an analytical solution has been derived [32], can be easily extended to those constitutive models and used to verify the solution found by the fully three-dimensional (3D) simulation code [9]. It is an indirect way of verifying the correctness of the implementation of the new method, but still it offers some degree of confidence because the simple 1D programs are easily control-

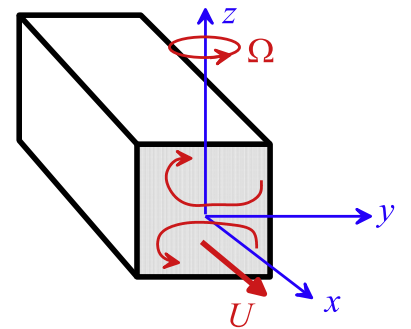

Fig. 1. Geometry for the rotating duct flow problem with indication of the expected secondary flow.

lable. This problem is then extended to the case of start-up flow in a duct of square cross-section, which is two-dimensional (2D) in space but without effective convection effects (since the flow is unidirectional and assumed fully developed in that direction). The existing analytical solution for the final steady state of a Newtonian fluid (e.g. [33]) is also valid for constant shear-viscosity viscoelastic fluids (such as the FENE-CR), and may thus serve to ascertain in part the present simulations. The last problem is much more complicate: we consider the start-up of flow in a rotating duct (Fig. 1). Coriolis acceleration will generate secondary flow in the duct cross-section, thus introducing convection effects in the problem, which although being 2D in space has all three velocity components and all six stress components as non-zero. It is an interesting flow problem, with some similarities to that of flow in curved ducts, and one which has never been tested with nonNewtonian viscoelastic fluids.

\section{Constitutive equations and method}

Any general time-dependent viscoelastic flow problem requires solution of the conservation equations for mass and momentum, and of a rheological equation for the extra stress tensor. The present numerical method is only effective for differential constitutive equations expressed in terms of the stress tensor, non-linear in the stress and of the FENE-P type (this qualification is clarified below). Since the FENE-P was developed for dilute polymer solutions, the extra stress possesses a Newtonian solvent contribution which is considered explicitly in the foregoing equations.

\subsection{Equation of motion}

Conservation of linear momentum is expressed as

$\rho \frac{D \mathbf{u}}{D t}=-\nabla p+\eta_{s} \nabla^{2} \mathbf{u}+\nabla \cdot \boldsymbol{\tau}+\mathbf{F}$

where the velocity vector is $\mathbf{u}=(u, v, w)$, the pressure $p, \eta_{s}$ is the solvent viscosity (assumed Newtonian) and $\boldsymbol{\tau}=\left(\tau_{i j}\right)$ the polymeric stress tensor. $\mathbf{F}$ represents a body force per unit volume (such as that due to gravitational acceleration) or others not included in the remaining terms; it is generally zero, except in the rotating duct case due to the pseudo-forces arising from a non-inertial reference frame (Section 4.3).

\subsection{Equation of continuity}

Conservation of mass, assuming an incompressible fluid with constant density $\rho$ :

$\nabla \cdot \mathbf{u}=0$

\subsection{Rheological models}

In addition to the Newtonian fluid $\left(\boldsymbol{\tau}_{s}=\eta_{s} \dot{\gamma}\right.$, when viewed as the solvent, and $\boldsymbol{\tau}=0$ in Eq. (1)), we consider the following three typical constitutive differential models: 
Table 1

Computational cost for start-up of channel flow: number of iterations required by the various constitutive models and solution methods.

\begin{tabular}{lllllll}
\hline & FENE-MCR & FENE-CR & & & FENE-P & \\
Mesh $101 \times 101$ & Method 1 & Method 1 & Method 2 & & Method 1 & Method 2 \\
\hline Total number iter. & 6142 & 24,069 & 5765 & & 33,769 & 6699 \\
Average n ${ }^{\circ}$ iter. & 3.07 & 12.03 & 2.88 & & 16.88 & 3.35 \\
Max. iter. per $\Delta t$ & 4 & 25 & 4 & & 29 & 5 \\
Iter. per $\Delta t$ at end & 3 & 5 & 2 & & 13 & 3 \\
\hline
\end{tabular}

\section{Model FENE-MCR [7]:}

$\boldsymbol{\tau}+\frac{\lambda}{f} \frac{D \boldsymbol{\tau}}{D t}=\eta_{p} \dot{\gamma}+\frac{\lambda}{f}\left(\boldsymbol{\tau} \cdot \nabla \mathbf{u}+\nabla \mathbf{u}^{T} \cdot \boldsymbol{\tau}\right)$

\section{Model FENE-CR [3]:}

$\boldsymbol{\tau}+\lambda \frac{D}{D t}\left(\frac{\boldsymbol{\tau}}{f}\right)=\eta_{p} \dot{\gamma}+\frac{\lambda}{f}\left(\boldsymbol{\tau} \cdot \nabla \mathbf{u}+\nabla \mathbf{u}^{T} \cdot \boldsymbol{\tau}\right)$

Model FENE-P [1,2]:

$\boldsymbol{\tau}+\lambda \frac{D}{D t}\left(\frac{\boldsymbol{\tau}}{f}\right)=\frac{a \eta_{p}}{f} \dot{\gamma}+\frac{\lambda}{f}\left(\boldsymbol{\tau} \cdot \nabla \mathbf{u}+\nabla \mathbf{u}^{T} \cdot \boldsymbol{\tau}\right)-\mathbf{I} \frac{D}{D t}\left(\frac{a \eta_{p}}{f}\right)$

In all models, the extensibility function is

$f=\frac{\left(L^{2}+\left(\lambda / a \eta_{p}\right) \operatorname{tr}(\boldsymbol{\tau})\right)}{\left(L^{2}-3\right)}$

and the following are constant parameters of the models: $\lambda$ - relaxation time (at zero deformation rate); $L^{2}$ - maximum extensibility (sometimes defined as $b=L^{2}-5$ ); $\eta_{p^{-}}$polymer contribution to the viscosity (at zero deformation rate; in Table 1 of Bird [34] an excellent review of kinetic theory of polymeric liquids - it is given $\eta_{p}=(b /(b+5)) n k T \lambda$ in terms of kinetic model parameters, which is consistent with our notation in Eq. (5) and the relationship $b=L^{2}-5$ ); $a$ - constant used for the FENE-P, with value $a=\frac{L^{2}}{L^{2}-3}=\frac{(b+5)}{(b+2)}$, and meaning $a=f_{\text {equil. }}$ (at equilibrium the polymer stress is zero, cf. [34] Eq. (16)). For the FENE-MCR and FENECR models there is no need to use the $a$ constant and we put $a=1$.

Collecting the above constitutive models in a single equation, we qualify as FENE-P type equations those we may write as:

$\boldsymbol{\tau}+\overbrace{\left(\frac{\lambda \boldsymbol{\tau}}{f}\right)}^{\nabla}=\eta_{p}^{\prime} \dot{\gamma}+\mathbf{g}(\boldsymbol{\tau}, \dot{\gamma}, \operatorname{Inv}(\boldsymbol{\tau}))$

where $\nabla$ is the Oldroyd upper-convected derivative [4], $\lambda$ is generally constant and $\eta_{p}^{\prime}$ is either the constant polymer contribution to the viscosity $\left(\eta_{p}\right)$ or a polymer viscosity function that depends on invariants of $\boldsymbol{\tau}$, as does the function $\mathbf{g}$ comprising terms such the substantial derivative of $\eta_{p} / f$ (in Eq. (5)) or those resulting from the lower-convected derivative (if present), or others from more sophisticated molecular based models.

\subsection{Reduced stress FENE-P model}

It is evident from Eqs. (5) (or (4)) and (6) that the time advancement of the stress components $\tau_{i j}$ should be accompanied by a simultaneous time advancement of the function $f$ which, in turn, depends on the stress components through the trace $\tau_{k k}$. Such inherent non-linearity cannot be accommodated by the usual temporal integration methods for the constitutive equations (like the Euler explicit or implicit, Crank-Nicolson, three time-level schemes, or variations thereof) and so the stresses in the function $f\left(\tau_{k k}\right)$ must lag the newly calculated stress in an iterative sequence giving rise to a slow-down of the simultaneous $\tau_{i j} / f$ time advancement procedure. Since $f\left(\tau_{k k}\right)$ is an increasing function of $\tau_{k k}$ (because $\tau_{k k}$ is always positive), when $\tau_{i j}$ increases with time the option of advancing $\tau_{i j} / f\left(\tau_{k k}^{*}\right)$ (where the asterisk in $\tau_{i j}^{*}$ indicates the value of $\tau_{i j}$ at the previous iteration) gives a smaller stress increment when compared to the simultaneous advancement of $\tau_{i j} / f\left(\tau_{k k}\right)$. As a consequence, more iterations will be necessary to bring the terms into balance, often implying an oscillating path to convergence. In the Appendix, a more quantitative argument is given for the deterioration of convergence rate when an approximate $1 \mathrm{D}$ version of the equation for $\tau_{x x}$ is advanced, instead of $\tau_{x x} / f\left(\tau_{x x}\right)$.

It appears therefore that convergence gains can be achieved if the quantity being advanced in time when the constitutive equations are integrated is $\tau_{i j} / f$ instead of $\tau_{i j}$. In addition, it turns out that the equations are only marginally affected by changing the dependent variable from $\tau_{i j}$ to $\tau_{i j} / f$, as it is now shown.

The reduced stress is defined by the ratio of the polymer stress to the extensibility function, as:

$\boldsymbol{\tau}^{\prime} \equiv \boldsymbol{\tau} / f$

Similarly, we may define a reduced polymer viscosity as:

$\eta_{p}^{\prime} \equiv a \eta_{p} / f$

being clear that this viscosity is variable, decreasing as the trace of the polymer stress increases.

These definitions are introduced into the FENE-P equation above, giving readily:

$f \cdot \boldsymbol{\tau}^{\prime}+\lambda \frac{D \boldsymbol{\tau}^{\prime}}{D t}=\eta_{p}^{\prime} \dot{\gamma}+\lambda\left(\boldsymbol{\tau}^{\prime} \cdot \nabla \mathbf{u}+\nabla \mathbf{u}^{T} \cdot \boldsymbol{\tau}^{\prime}\right)-\mathbf{I} \frac{D \eta_{p}^{\prime}}{D t}$

while a simple manipulation of the extensibility functions allows it to be re-written in terms of $\boldsymbol{\tau}^{\prime}$ as:

$f=\frac{L^{2}}{\left(L^{2}-3-\left(\lambda / a \eta_{p}\right) \operatorname{tr}\left(\boldsymbol{\tau}^{\prime}\right)\right)}$

These are the equations for the reduced FENE-P model, to be implemented numerically in what we shall denote "method $2^{\prime \prime}$, while the method for the original FENE-P equation will be referred to as "method 1" (that used in previous publications, eg, [18]).

It is instructive to divide the reduced FENE-P Eq. (10) by the extensibility functions so that it becomes analogous to the FENEMCR equation:

$\boldsymbol{\tau}^{\prime}+\frac{\lambda}{f} \frac{D \boldsymbol{\tau}^{\prime}}{D t}=\frac{\eta_{p}^{\prime}}{f} \dot{\gamma}+\frac{\lambda}{f}\left(\boldsymbol{\tau}^{\prime} \cdot \nabla \mathbf{u}+\nabla \mathbf{u}^{T} \cdot \boldsymbol{\tau}^{\prime}\right)-\mathbf{I} \frac{1}{f} \frac{D}{D t}\left(\eta_{p}^{\prime}\right)$

Clearly, if we make the identifications $\boldsymbol{\tau}^{\prime} \Leftrightarrow \boldsymbol{\tau}$ and $\eta_{p}^{\prime} \Leftrightarrow \eta_{p}$, the two Eqs. (3) and (12) have similar form, except for the last term, which is specific to the FENE-P model, and the fact that in this model the viscosity is shear-thinning (seen here in the division of the polymer viscosity term - that multiplied by the shear rate by the function $f$ ). So, we hope that the numerical behaviour of the new reduced-stress method will be akin to that of the standard method with the FENE-MCR model, which, as we have already commented, is the easiest in respect to numerical stability and efficiency. 


\subsection{Simplified equations for $1 D$ flow case}

To make the differences between methods easier to follow and to facilitate the explanation of the numerical procedure of the next section, we restrict the general equations given above in vector form to the 1D flow case corresponding to start-up of channel flow.

For this problem, of fully developed flow in a channel lying on the $(x, y)$ plane, subject to a constant pressure gradient $P=$ $-\partial p / \partial x$, the only non-zero velocity component is $u$, which varies with $y$ and $t$, and the only non-zero stress components are $\tau_{x y}$ (shear) and $\tau_{x x}$ (normal) (although for the FENE-P the transversal, or secondary, normal components, $\tau_{y y}$ e $\tau_{z z}$, are also non-zero and equal to each other).

Using the FENE-P as representative rheological model, since the others may be obtained by appropriate simplifications, the equations above (Eqs. (1), ( 5) and (6)) reduce to:

$\rho \frac{\partial u}{\partial t}=P+\eta_{s} \frac{\partial^{2} u}{\partial y^{2}}+\frac{\partial \tau_{x y}}{\partial y}$

$\tau_{x y}+\lambda \frac{\partial}{\partial t}\left(\frac{\tau_{x y}}{f}\right)=\frac{a \eta_{p}}{f} \frac{\partial u}{\partial y}+\frac{\lambda p}{f} \frac{\partial u}{\partial y} \tau_{y y}$

$\tau_{x x}+\lambda \frac{\partial}{\partial t}\left(\frac{\tau_{x x}}{f}\right)=2 \lambda \frac{\tau_{x y}}{f} \frac{\partial u}{\partial y}-a \eta_{p} \frac{\partial}{\partial t}\left(\frac{1}{f}\right)$

$\tau_{y y}+\lambda \frac{\partial}{\partial t}\left(\frac{\tau_{y y}}{f}\right)=-a \eta_{p} \frac{\partial}{\partial t}\left(\frac{1}{f}\right) ; \tau_{z z}=\tau_{y y}$

and function

$f=\left(L^{2}+\left(\lambda / a \eta_{p}\right) \tau_{k k}\right) /\left(L^{2}-3\right)$

with

$\tau_{k k}=\operatorname{tr}(\boldsymbol{\tau})=\tau_{x x}+\tau_{y y}+\tau_{z z}=\tau_{x x}+2 \tau_{y y}$.

It is noted that the velocity is only directly affected by the shear component of the polymeric stress $\tau_{x y}$, but the normal components $\tau_{x x}$ and $\tau_{y y}=\tau_{z z}$ affect $\tau_{x y}$ through the function $f=f\left(\tau_{k k}\right)$ (for the FENE-MCR and CR, we have $\tau_{k k}=\tau_{x x}$ ). In the case of the FENE-P, $\tau_{y y}$ has also a direct influence on $\tau_{x y}$, last term of Eq. (13b) (but not in the case of the FENE-MCR and CR, since $\tau_{y y}=0$ ).

Now, using the reduced-stress the equation of motion (Eq. (13a)) remains unaltered and the constitutive equations become:

$f \tau^{\prime}{ }_{x y}+\lambda \frac{\partial \tau_{x y}^{\prime}}{\partial t}=\frac{a \eta_{p}}{f} \frac{\partial u}{\partial y}+\lambda \tau^{\prime}{ }_{y y} \frac{\partial u}{\partial y}$

$f \tau_{x x}^{\prime}+\lambda \frac{\partial \tau_{x x}^{\prime}}{\partial t}=2 \lambda \tau_{x y}^{\prime} \frac{\partial u}{\partial y}-a \eta_{p} \frac{\partial}{\partial t}\left(\frac{1}{f}\right)$

$f \tau^{\prime}{ }_{y y}+\lambda \frac{\partial \tau_{y y}^{\prime}}{\partial t}=-a \eta_{p} \frac{\partial}{\partial t}\left(\frac{1}{f}\right)$

$f=f\left(\tau^{\prime}{ }_{k k}\right)=L^{2} /\left(L^{2}-3-\left(\lambda / a \eta_{p}\right) \tau^{\prime}{ }_{k k}\right)$.

\subsection{Non-dimensional variables and parameters}

Dimensionless parameters relevant to the present flow problems, with a channel or a duct half-height $H$, an average velocity in steady state $U$, and a pressure gradient $P$, are:

- elasticity, elastic number $E=\eta_{0} \lambda / \rho H^{2}$;

- elasticity (alternative), Deborah number $D e=\lambda U / H$;

- extensibility $L^{2}$ (or $b=L^{2}-5$ );

- polymer concentration, $\beta=\eta_{s} / \eta_{0}$ (with $\eta_{0}=\eta_{s}+\eta_{p}$ ).

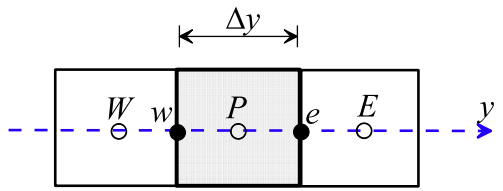

Fig. 2. Representation of a $1 \mathrm{D}$ cell and notation for neighbouring cells.

Although the previous equations are dimensional, they may be viewed as non-dimensional by assigning: $\rho=1 ; \eta_{0}=1 ; \eta_{p}=$ $1-\beta ; \eta_{s}=\beta ; U=1 ; H=1 ; \lambda=E$ and $P=3$ (for constant viscosity channel flow, using Poiseuille's law $\left.U=P H^{2} / 3 \mu\right)$. Time is then normalized with a diffusion time scale, $t \equiv t /\left(\rho H^{2} / \eta_{0}\right)$; velocity as $u \equiv u / U$; and stresses as $\tau_{i j} \equiv \tau_{i j} /\left(\eta_{0} U / H\right)$. For the constant viscosity models (Newtonian, FENE-MCR and FENE-CR) the value of the constant pressure gradient is chosen so that the average velocity becomes unity and, in this case, we have also $\lambda=D e$. However, for the FENE-P model whose shear viscosity in steady state is shearthinning $(\eta(\dot{\gamma})$ decreases as the shear-rate $\dot{\gamma}$ increases), when we put $P=3$ in a channel flow the average velocity in steady state becomes larger than unity $(U>1)$, and so the effective Deborah number will be larger than the imposed Deborah number (we will have $D e \equiv D e \times U)$. When appropriate those differences are explicitly indicated.

\section{Discretization and numerical method}

Discretization of the differential equations and explanation of the numerical method, of the finite volume type, are here described with some detail for the simplest problem of start-up of fully-developed channel flow.

For this simple problem, velocity and stress vary with time $t$ and the lateral spatial coordinate $y$. Discretization equations, the algebraic counterpart of the differential ones, are obtained by integrating the differential equations of the previous section over a control volume $\Delta y$ and over a time step $\Delta t$. Spatial integration is based on central differences and on the middle-point rule (both of 2nd order accuracy). Temporal integration is based on either the Euler implicit method (of 1st order accuracy) or the threetime level method (of 2nd order accuracy). The former is employed in the present description, to avoid unnecessary complications at this point, while the latter is actually used in the computations. Time levels are indicated by indices $n$ (present time) and $n+1$ (future time, to be determined), while the time step is here assumed constant $\Delta t=t^{n+1}-t^{n}$. Since the temporal integration is fully implicit, all terms in the right-hand side of the differential equations will be approximated at the new time level $n+1$, which implies the need for iteration (the outer iterations, to distinguish from the inner iterations in the iterative linear-equation solver). Spatial positions follow the usual notation for control volumes in the finite volume method (see Fig. 2): central node $P$, node to the right $E$ ("East"), node to the left $W$ ("West"); positions at the faces of the control volume centred at $P$ are indicated by small lettering $e$ and $w$.

\subsection{Equation of motion}

Straightforward integration of Eq. (13a) with the Euler scheme and central differences gives:

$$
\begin{aligned}
\frac{\rho \Delta y}{\Delta t}\left(u_{P}^{n+1}-u_{P}^{n}\right)= & P+\left(\eta_{s} \frac{u_{E}^{n+1}-u_{P}^{n+1}}{\Delta y}\right)-\left(\eta_{s} \frac{u_{P}^{n+1}-u_{W}^{n+1}}{\Delta y}\right) \\
& +\left(\tau_{x y}\right)_{e}^{n+1}-\left(\tau_{x y}\right)_{w}^{n+1}
\end{aligned}
$$

which is written under standard form as:

$a_{P} u_{P}^{n+1}=a_{E} u_{E}^{n+1}+a_{W} u_{W}^{n+1}+b$ 
with coefficients:

$a_{E}=\eta_{s} / \Delta y ; a_{W}=\eta_{s} / \Delta y ; a_{P}^{n}=\rho \Delta y / \Delta t ; a_{P}=a_{E}+a_{W}+a_{P}^{n}$

and source term:

$b=a_{P}^{n} u_{P}^{n}+P+\left[\left(\tau_{x y}\right)_{e}^{n+1}-\left(\tau_{x y}\right)_{w}^{n+1}\right]$

The set of equations represented by Eq. (15a) is tri-diagonal and may be solved with the TDMA algorithm (for this simple problem, there is no need for inner iterations: the TDMA acts as a direct solver). Boundary conditions for the velocity are assigned by assuming no-slip at walls, $y=-H$ and $y=+H$. Stress components at the faces of control volumes, which arise from the divergence source term $\nabla \cdot \boldsymbol{\tau} \equiv \Delta \tau_{x y, P}=\tau_{x y, e}-\tau_{x y, w}$, are calculated with the usual technique [9] (for any face $f$ ):

$\left(\tau_{x y}\right)_{f}={\overline{\left(\tau_{x y}\right)_{f}}}+\eta_{p} \frac{\Delta u_{f}}{\Delta y} \overline{\left(\frac{1}{a_{P}^{\tau} / \Delta y}\right)_{f}}-\overline{\left(\frac{\eta_{p} \Delta u_{P}}{a_{P}^{\tau}}\right)_{f}}$

Average values, indicated by the over-line, are obtained by linear interpolation (in the 1D codes, with uniform mesh, this is the same as doing arithmetical averaging). Boundary conditions are also required in the momentum equation for the stresses at the walls; a local Couette approximation is not viable in unsteady flows [31] and we use here instead either the approach described in that reference or a simpler alternative: the stress components are linearly extrapolated, from the two closer adjacent nodes, to the wall (Prof. M.A. Alves, personal communication).

\subsection{FENE-P equation}

In the absence of convective terms, the stress components at control volume $P$ are disconnected from the stresses at the surrounding control volumes. The solution of the equations can then be obtained by successive substitution, without the need of calling the tri-diagonal solver.

\subsection{Method 1} gives:

Integration of Eqs. (13b)-(13d) with the implicit Euler method

$$
\begin{aligned}
& \Delta y\left(\tau_{x y}\right)_{P}^{n+1}+\frac{\lambda \Delta y}{\Delta t}\left(\frac{\left(\tau_{x y}\right)_{P}^{n+1}}{f_{P}^{n+1}}-\frac{\left(\tau_{x y}\right)_{P}^{n}}{f_{P}^{n}}\right) \\
& =\frac{a \eta_{p}}{f_{P}^{n+1}}\left(u_{e}^{n+1}-u_{w}^{n+1}\right)+\frac{\lambda}{f_{P}^{n+1}}\left(\tau_{y y}\right)_{P}^{n+1}\left(u_{e}^{n+1}-u_{w}^{n+1}\right) \\
& \Delta y\left(\tau_{x x}\right)_{P}^{n+1}+\frac{\lambda \Delta y}{\Delta t}\left(\frac{\left(\tau_{x x}\right)_{P}^{n+1}}{f_{P}^{n+1}}-\frac{\left(\tau_{x x}\right)_{P}^{n}}{f_{P}^{n}}\right) \\
& =2 \frac{\lambda}{f_{P}^{n+1}} \tau_{x y, P}^{n+1}\left(u_{e}^{n+1}-u_{w}^{n+1}\right)-\frac{a \eta_{p} \Delta y}{\Delta t}\left(\frac{1}{f_{P}^{n+1}}-\frac{1}{f_{P}^{n}}\right) \\
& \Delta y\left(\tau_{y y}\right)_{P}^{n+1}+\frac{\lambda \Delta y}{\Delta t}\left(\frac{\left(\tau_{y y}\right)_{P}^{n+1}}{f_{P}^{n+1}}-\frac{\left(\tau_{y y}\right)_{P}^{n}}{f_{P}^{n}}\right)=-\frac{a \eta_{p} \Delta y}{\Delta t}\left(\frac{1}{f_{P}^{n+1}}-\frac{1}{f_{P}^{n}}\right)
\end{aligned}
$$

and the extensibility function (Eq. (13e)) is evaluated as:

$f_{P}^{n+1}=\frac{\left(L^{2}+\left(\frac{\lambda}{a \eta_{p}}\right)\left(\left(\tau_{x x}\right)_{P}^{n+1}+2\left(\tau_{y y}\right)_{P}^{n+1}\right)\right)}{\left(L^{2}-3\right)}$.

These equations are re-written under standard discretization form as:

$a_{P}^{\tau}\left(\tau_{x y}\right)_{P}^{n+1}=b^{\tau_{x y}}+a_{P}^{\tau, n}\left(\tau_{x y}\right)_{P}^{n}$

$a_{P}^{\tau}\left(\tau_{x x}\right)_{P}^{n+1}=b^{\tau_{x x}}+a_{P}^{\tau, n}\left(\tau_{x x}\right)_{P}^{n}$
$a_{P}^{\tau}\left(\tau_{y y}\right)_{P}^{n+1}=b^{\tau_{y y}}+a_{P}^{\tau, n}\left(\tau_{y y}\right)_{P}^{n}$

with coefficients and source terms:

$$
\begin{aligned}
a_{P}^{\tau}=\Delta y\left(1+\frac{\lambda}{f_{P}^{n+1} \Delta t}\right) \\
a_{P}^{\tau, n}=\Delta y\left(\frac{\lambda}{f_{P}^{n} \Delta t}\right) \\
b^{\tau_{x y}}=\frac{a \eta_{p}}{f_{P}^{n+1}}\left(u_{e}^{n+1}-u_{w}^{n+1}\right)+\frac{\lambda}{f_{P}^{n+1}}\left(\tau_{x y}\right)_{P}^{n+1}\left(u_{e}^{n+1}-u_{w}^{n+1}\right) \\
b^{\tau_{x x}}=\frac{2 \lambda\left(\tau_{x y}\right)_{P}^{n+1}}{f_{P}^{n+1}}\left(u_{e}^{n+1}-u_{w}^{n+1}\right)-\frac{a \eta_{p} \Delta y}{\Delta t}\left(\frac{1}{f_{P}^{n+1}}-\frac{1}{f_{P}^{n}}\right) \\
b^{\tau_{y y}}=-\frac{a \eta_{p} \Delta y}{\Delta t}\left(\frac{1}{f_{P}^{n+1}}-\frac{1}{f_{P}^{n}}\right)
\end{aligned}
$$

The iteration cycle carried out inside each time step, here written in a simplified form (when not specified, variables are evaluated at the central node $P$ ), becomes:

$$
\begin{aligned}
& {\left[\left(\frac{\rho \Delta y}{\Delta t}+a_{E}+a_{W}\right) u_{P}^{*}=a_{E} u_{E}^{*}+a_{W} u_{W}^{*}+P+\Delta \tau_{x y, P}^{*}+\frac{\rho \Delta y}{\Delta t} u_{P}^{n}\right.} \\
& f^{*}=\frac{\left(L^{2}+\left(\lambda / a \eta_{p}\right)\left(\tau_{x x}^{*}+2 \tau_{y y}^{*}\right)\right)}{\left(L^{2}-3\right)} \\
& \Delta y\left(1+\frac{\lambda}{f^{*} \Delta t}\right) \tau_{y y}^{*}=\frac{\lambda \Delta y}{f^{n} \Delta t} \tau_{y y}^{n}-\frac{a \eta_{p} \Delta y}{\Delta t}\left(\frac{1}{f^{*}}-\frac{1}{f^{n}}\right) \\
& \Delta y\left(1+\frac{\lambda}{f^{*} \Delta t}\right) \tau_{x y}^{*}=\frac{a \eta_{p}}{f^{*}} \Delta u_{P}^{*}+\frac{\lambda}{f^{*}} \tau_{y y}^{*} \Delta u_{P}^{*}+\frac{\lambda \Delta y}{f^{n} \Delta t} \tau_{x y}^{n} \\
& \underbrace{\Delta y\left(1+\frac{\lambda}{f^{*} \Delta t}\right)}_{a_{P}^{\tau}} \tau_{x x}^{*}=2 \lambda \frac{\tau_{x y}^{*}}{f^{*}} \Delta u_{P}^{*}+\frac{\lambda \Delta y}{f^{n} \Delta t} \tau_{x x}^{n}-\frac{a \eta_{p} \Delta y}{\Delta t}\left(\frac{1}{f^{*}}-\frac{1}{f^{n}}\right) \\
& \tau_{x y, e}^{*}=\overline{\tau_{x y_{e}}^{*}}+\frac{\Delta u_{e}^{*}}{\Delta y} \overline{\left(\frac{\eta_{p}^{\prime}}{a_{P}^{\tau} / \Delta y}\right)_{e}}-\overline{\left(\frac{\eta_{p}^{\prime} \Delta u_{P}^{*}}{a_{P}^{\tau}}\right)_{e}}\left(\text { with } \eta_{p}^{\prime}=a \eta_{p} / f^{*}\right)
\end{aligned}
$$

In the equation of motion, the stress difference is $\Delta \tau_{x y, P}^{*}=$ $\tau_{x y, e}^{*}-\tau_{x y, w}^{*}$, where the shear stress components at cell faces are calculated with the special interpolation method [9] given in the last line. In the stress equations, the difference of velocities is $\Delta u_{p}^{*}=\bar{u}_{e}^{*}-\bar{u}_{w}^{*}$, where velocities at cell faces are obtained by linear interpolation, for example $\bar{u}_{e}^{*}=0.5\left(u_{P}^{*}+u_{E}^{*}\right)$.

The order of solution of the several stress equations is chosen so that the source terms may be evaluated with the most recent stress values: first we calculate $\tau_{y y}$ whose value goes immediately to the next equation to be solved, for $\tau_{x y}$; the last equation is for $\tau_{x x}$ which possesses a source term in $\tau_{x y}$. After having calculated the stresses at all nodal locations, we apply the special interpolation for the cell faces; the central coefficient of the stress equations is $a_{p}^{\tau}=\Delta y\left(1+\lambda /\left(f^{*} \Delta t\right)\right)$ or, in intensive form, $a_{p}^{\tau} / \Delta y=$ $\left(1+\lambda /\left(f^{*} \Delta t\right)\right)$. The difference of velocities at e.g. the "east" face is $\Delta u_{e}^{*}=u_{E}^{*}-u_{P}^{*}$, which is evaluated directly at the face without the need of any interpolation. The two last terms in the expression for the shear stress at the "east" face represent the difference between the interpolated velocity gradient and the one evaluated directly at the face; they prevent the occurrence of stress oscillations. With a uniform mesh, the $\Delta y$ in the middle term may be included in the linear interpolation and the final expression becomes simpler, with $a_{p}^{\tau}$ in the denominator instead of $a_{p}^{\tau} / \Delta y$. 
The iterative cycle inside each time step is repeated until the larger residual of all equations becomes smaller than a given tolerance. Residuals are evaluated as:

$\operatorname{res}(u)=\sum_{P}\left|a_{P} u_{P}^{*}-\left(a_{E} u_{E}^{*}+a_{W} u_{W}^{*}+P+\Delta \tau_{x y, P}^{*}+\frac{\rho \Delta y}{\Delta t} u_{P}^{n}\right)\right|$

$$
\begin{aligned}
\operatorname{res}\left(\tau_{x y}\right)= & \sum_{P} \mid \Delta y\left(1+\frac{\lambda}{f^{*} \Delta t}\right) \tau_{x y}^{*} \\
& -\left(\frac{a \eta_{p}}{f^{*}} \Delta u_{P}^{*}+\frac{\lambda}{f^{*}} \tau_{y y}^{*} \Delta u_{P}^{*}+\frac{\lambda \Delta y}{f^{n} \Delta t} \tau_{x y}^{n}\right) \mid \\
\operatorname{res}\left(\tau_{x x}\right)= & \sum_{P} \mid \Delta y\left(1+\frac{\lambda}{f^{*} \Delta t}\right) \tau_{x x}^{*} \\
& -\left[2 \lambda \frac{\tau_{x y}^{*}}{f^{*}} \Delta u_{P}^{*}+\frac{\lambda \Delta y}{f^{n} \Delta t} \tau_{x x}^{n}-\frac{a \eta_{p} \Delta y}{\Delta t}\left(\frac{1}{f^{*}}-\frac{1}{f^{n}}\right)\right] \mid
\end{aligned}
$$

$$
\begin{aligned}
\operatorname{res}\left(\tau_{y y}\right)= & \sum_{P} \mid \Delta y\left(1+\frac{\lambda}{f^{*} \Delta t}\right) \tau_{y y}^{*} \\
& -\left[\frac{\lambda \Delta y}{f^{n} \Delta t} \tau_{y y}^{n}-\frac{a \eta_{p} \Delta y}{\Delta t}\left(\frac{1}{f^{*}}-\frac{1}{f^{n}}\right)\right] \mid
\end{aligned}
$$

resid $=\max \left\{\operatorname{res}(u), \operatorname{res}\left(\tau_{x y}\right), \operatorname{res}\left(\tau_{x x}\right), \operatorname{res}\left(\tau_{y y}\right)\right\} \Rightarrow$ resid $<T O L$

where the sums are carried out over all internal grid points at the centres of the control volumes forming the mesh. After having achieved iterative convergence, we assign the converged values to the new time level $u_{P}^{n+1}=u_{P}^{*}, \tau_{x y, P}^{n+1}=\tau_{x y, P}^{*}, \tau_{x x, P}^{n+1}=\tau_{x x, P}^{*}$ and $\tau_{y y, P}^{n+1}=\tau_{y y, P}^{*}$, and proceed to the next time level $(n \rightarrow n+1)$.

\subsection{Method 2} gives:

Integration of the reduced-stress equations (Eqs. (14a)-(14c))

$$
\begin{aligned}
& \Delta y f_{P}^{n+1}\left(\tau_{x y}^{\prime}\right)_{P}^{n+1}+\frac{\lambda \Delta y}{\Delta t}\left(\left(\tau_{x y}^{\prime}\right)_{P}^{n+1}-\left(\tau_{x y}^{\prime}\right)_{P}^{n}\right) \\
& =\frac{a \eta_{p}}{f_{P}^{n+1}}\left(u_{e}^{n+1}-u_{w}^{n+1}\right)+\lambda\left(\tau^{\prime}{ }_{y y}\right)_{P}^{n+1}\left(u_{e}^{n+1}-u_{w}^{n+1}\right) \\
& \Delta y f_{P}^{n+1}\left(\tau_{x x}^{\prime}\right)_{P}^{n+1}+\frac{\lambda \Delta y}{\Delta t}\left(\left(\tau_{x x}^{\prime}\right)_{P}^{n+1}-\left(\tau^{\prime}{ }_{x x}\right)_{P}^{n}\right) \\
& =2 \lambda \tau_{x y, P}^{\prime n+1}\left(u_{e}^{n+1}-u_{w}^{n+1}\right)-\frac{a \eta_{p} \Delta y}{\Delta t}\left(\frac{1}{f_{P}^{n+1}}-\frac{1}{f_{P}^{n}}\right) \\
& \Delta y f_{P}^{n+1}\left(\tau_{y y}^{\prime}\right)_{P}^{n+1}+\frac{\lambda \Delta y}{\Delta t}\left(\left(\tau_{y y}^{\prime}\right)_{P}^{n+1}-\left(\tau^{\prime}{ }_{y y}\right)_{P}^{n}\right) \\
& =-\frac{a \eta_{p} \Delta y}{\Delta t}\left(\frac{1}{f_{P}^{n+1}}-\frac{1}{f_{P}^{n}}\right)
\end{aligned}
$$

and an extensibility function,

$$
f_{P}^{n+1}=\frac{L^{2}}{\left(L^{2}-3-\left(\frac{\lambda}{a \eta_{p}}\right)\left(\left(\tau_{x x}^{\prime}\right)_{P}^{n+1}+2\left(\tau_{y y}^{\prime}\right)_{P}^{n+1}\right)\right)}
$$

which are re-written under standard form:

$$
\begin{aligned}
& a_{P}^{\tau^{\prime}}\left(\tau^{\prime}{ }_{x y}\right)_{P}^{n+1}=b^{\tau^{\prime}{ }_{x y}}+a_{P}^{\tau^{\prime}, n}\left(\tau^{\prime}{ }_{x y}\right)_{P}^{n} \\
& a_{P}^{\tau^{\prime}}\left(\tau^{\prime}{ }_{x x}\right)_{P}^{n+1}=b^{\tau^{\prime}{ }_{x x}}+a_{P}^{\tau^{\prime}, n}\left(\tau^{\prime}{ }_{x x}\right)_{P}^{n}
\end{aligned}
$$

$a_{P}^{\tau^{\prime}}\left(\tau^{\prime}{ }_{y y}\right)_{P}^{n+1}=b^{\tau^{\prime} y}+a_{P}^{\tau^{\prime}, n}\left(\tau_{y y}^{\prime}\right)_{P}^{n}$

with coefficients and source terms:

$a_{P}^{\tau^{\prime}}=\Delta y\left(f_{P}^{n+1}+\frac{\lambda}{\Delta t}\right)$

$a_{P}^{\tau^{\prime}, n}=\frac{\lambda \Delta y}{\Delta t}$

$b^{\tau^{\prime} x y}=\frac{a \eta_{p}}{f_{P}^{n+1}}\left(u_{e}^{n+1}-u_{w}^{n+1}\right)+\lambda\left(\tau^{\prime}{ }_{y y}\right)_{P}^{n+1}\left(u_{e}^{n+1}-u_{w}^{n+1}\right)$

$b^{\tau^{\prime} x x}=2 \lambda\left(\tau_{x y}^{\prime}\right)_{P}^{n+1}\left(u_{e}^{n+1}-u_{w}^{n+1}\right)-\frac{a \eta_{p} \Delta y}{\Delta t}\left(\frac{1}{f_{P}^{n+1}}-\frac{1}{f_{P}^{n}}\right)$

$b^{\tau^{\prime} y y}=-\frac{a \eta_{p} \Delta y}{\Delta t}\left(\frac{1}{f_{P}^{n+1}}-\frac{1}{f_{P}^{n}}\right)$

The iterative cycle within each time step is now:

$$
\begin{aligned}
& {\left[\left(\frac{\rho \Delta y}{\Delta t}+a_{E}+a_{W}\right) u_{P}^{*}=a_{E} u_{E}^{*}+a_{W} u_{W}^{*}+P+\Delta \tau_{x y, P}^{*}+\frac{\rho \Delta y}{\Delta t} u_{P}^{n}\right.} \\
& f^{*}=\frac{L^{2}}{\left(L^{2}-3-\left(\lambda / a \eta_{p}\right)\left(\tau_{x x}^{\prime *}+2 \tau_{y y}^{\prime *}\right)\right)} \\
& \Delta y\left(f^{*}+\frac{\lambda}{\Delta t}\right) \tau_{y y}^{\prime *}=\frac{\lambda \Delta y}{\Delta t} \tau_{y y}^{\prime n}-\frac{a \eta_{p} \Delta y}{\Delta t}\left(\frac{1}{f^{*}}-\frac{1}{f^{n}}\right) \\
& \Delta y\left(f^{*}+\frac{\lambda}{\Delta t}\right) \tau_{x y}^{\prime *}=\frac{a \eta_{p}}{f^{*}} \Delta u_{P}^{*}+\lambda \tau_{y y}^{\prime *} \Delta u_{P}^{*}+\frac{\lambda \Delta y}{\Delta t} \tau_{x y}^{\prime n} \\
& \underbrace{\Delta y\left(f^{*}+\frac{\lambda}{\Delta t}\right)}_{a_{P}^{\tau^{\prime}}} \tau_{x x}^{\prime *}=2 \lambda \tau_{x y}^{\prime *} \Delta u_{P}^{*}+\frac{\lambda \Delta y}{\Delta t} \tau_{x x}^{\prime \prime}-\frac{a \eta_{p} \Delta y}{\Delta t}\left(\frac{1}{f^{*}}-\frac{1}{f^{n}}\right) \\
& \tau_{x y, e}^{*}=\overline{f \tau_{x y}^{\prime_{e}}}+a \eta_{p} \frac{\Delta u_{e}^{*}}{\Delta y} \overline{\left(\frac{1}{\left(a_{P}^{\tau^{\prime}} / f^{*}\right) / \Delta y}\right)_{e}}-\overline{\left(\frac{a \eta_{p} \Delta u_{P}^{*}}{a_{P}^{\tau^{\prime}} / f^{*}}\right)_{e}}
\end{aligned}
$$

Notice that the equation of motion is the same as in method 1 , with the stress divergence term based on the usual stress components $\tau_{x y}$ (evaluated at the control volume faces), while the constitutive equations are expressed in terms of the modified stresses $\tau_{x y}^{\prime}=\tau_{x y} / f, \tau_{x x}^{\prime}=\tau_{x x} / f$ e $\tau_{y y}^{\prime}=\tau_{y y} / f$. The central coefficient in the stress equations is now that of method 1 multiplied by the function $f$, that is $\left(a_{P}^{\tau}\right)_{\text {previous }}=\left(a_{P}^{\tau}\right)_{\text {now }} / f^{*}$.

\subsection{Three-time level scheme}

As mentioned above, for the sake of improved accuracy the temporal discretization employed in the actual computations is based on the three-time level scheme [35, Table 8.I, pp. 190], which is here implemented in a slightly modified version, allowing for variable time-step sizes. Any of the previous governing equations (Eq. (13)) may be written as

$\frac{\partial \phi}{\partial t}=H(\phi)$

where $\phi$ stands for the dependent variable, either a velocity or a stress component, and $H(\phi)$ is an operator comprising convective and diffusive terms, and the remaining source terms if they exist. With a fully-implicit temporal discretization, this equation is approximated as:

$\left(\frac{\partial \phi}{\partial t}\right)^{n+1}=H\left(\phi^{n+1}\right) \equiv H^{n+1}$ 
and the time derivative at the new time level $t^{n+1}$ is obtained by linear extrapolation of its value at the two precedent time levels, $t^{n+\frac{1}{2}}$ and $t^{n-\frac{1}{2}}$, as:

$$
\frac{(\partial \phi / \partial t)^{n+1}-(\partial \phi / \partial t)^{n+\frac{1}{2}}}{\Delta t^{n} / 2}=\frac{(\partial \phi / \partial t)^{n+\frac{1}{2}}-(\partial \phi / \partial t)^{n-\frac{1}{2}}}{\left(\Delta t^{n}+\Delta t^{n-1}\right) / 2}
$$

where varying the time steps are defined as $\Delta t^{n}=t^{n+1}-t^{n}$ and $\Delta t^{n-1}=t^{n}-t^{n-1}$. With the time-step ratio denoted as $r=$ $\Delta t^{n} / \Delta t^{n-1}$, this formula is re-arranged to

$(\partial \phi / \partial t)^{n+1}=(1+r /(1+r))(\partial \phi / \partial t)^{n+\frac{1}{2}}-(r /(1+r))(\partial \phi / \partial t)^{n-\frac{1}{2}}$ and after approximating the derivatives with central differences in time (2nd order accurate),

$$
\begin{aligned}
(\partial \phi / \partial t)^{n+1}= & (1+r /(1+r))\left(\frac{\phi^{n+1}-\phi^{n}}{\Delta t^{n}}\right) \\
& -(r /(1+r))\left(\frac{\phi^{n}-\phi^{n-1}}{\Delta t^{n-1}}\right)
\end{aligned}
$$

we arrive at the final expression for the three-time level scheme:

$(\partial \phi / \partial t)^{n+1}=\frac{(1+r /(1+r)) \phi^{n+1}-(1+r) \phi^{n}+r^{2} /(1+r) \phi^{n-1}}{\Delta t^{n}}$.

This expression should be substituted for the simpler Euler approximation $(\partial \phi / \partial t)^{n+1}=\left(\phi^{n+1}-\phi^{n-1}\right) / \Delta t$ used in the previous sub-sections. It is useful to see how it simplifies in some limiting cases. For a constant time-step interval, $r=1$, we obtain:

$(\partial \phi / \partial t)^{n+1}=\frac{\frac{3}{2} \phi^{n+1}-2 \phi^{n}+\frac{1}{2} \phi^{n-1}}{\Delta t}$

which is the usual expression given for the three-time level method in standard references $[18,19,28-30,35]$. When the time step is halved $(r=1 / 2)$, as occurs when convergence within a $\Delta t$ cannot be achieved, we have

$(\partial \phi / \partial t)^{n+1}=\frac{\frac{4}{3} \phi^{n+1}-\frac{3}{2} \phi^{n}+\frac{1}{6} \phi^{n-1}}{\Delta t^{n}}$

and when the time step is doubled $(r=2)$, as occurs when the temporal variation of the solution is smooth,

$(\partial \phi / \partial t)^{n+1}=\frac{\frac{5}{3} \phi^{n+1}-3 \phi^{n}+\frac{4}{3} \phi^{n-1}}{\Delta t^{n}}$

The larger the factor multiplying $\phi^{n+1}$ the better, as the diagonal dominance of the corresponding matrix equation is thus increased; this is the case for $r=2$. However, the larger the coefficient of $\phi^{n-1}$ the more problematic is the scheme, since this term is negative when moved to the right-hand side of the discretization equation and this impedes stability; the worst situation is again $r=2$.

In practice, it is useful to blend the above three-time level (3TL) scheme with the first-order implicit Euler scheme, using a blending factor $0 \leq \kappa \leq 1$, such that:

$(\partial \phi / \partial t)^{n+1}=\kappa(\partial \phi / \partial t)_{3 T L}^{n+1}+(1-\kappa)(\partial \phi / \partial t)_{\text {Euler }}^{n+1}$

The expression for the time derivative after applying such blending becomes:

$(\partial \phi / \partial t)^{n+1}=\frac{\left(1+\kappa \frac{r}{1+r}\right) \phi^{n+1}-(1+\kappa r) \phi^{n}+\kappa \frac{r^{2}}{1+r} \phi^{n-1}}{\Delta t^{n}}$

which is the actual expression implemented in the multidimensional code. For example, at the start of the flow, the $\phi^{n-1}$ are unknown and the Euler scheme must be used, by setting $\kappa=0$. Normally, second order accuracy in time is desirable and the full three-time level should be switched on, with $\kappa=1$.

\section{Results}

The algorithm explained in the previous section was implemented in both our 3D multidimensional general code and in several small FORTRAN codes which solve the 1D startup flow in channels, for each specific constitutive model. These 1D codes are similar among them and essentially follow the algorithm employed in the $3 \mathrm{D}$ code, thus serving to assess the results in a controlled manner.

As base values for the rheological parameters, often employed in numerical simulation studies (see [18] and references therein), we consider a typical extensibility parameter of $L^{2}=100$, a solvent viscosity ratio of $\beta=0.1$ (in the assessment of numerical methods and benchmark problems a value often used is $\beta=1 / 9$ ) and a relatively high elasticity number $E=5$. In most runs the numerical grid was uniform with either 51 or 101 internal control volumes along each direction; an odd number of nodes is chosen so that a control volume is situated exactly at the central point of the channel or the square cross-section duct. On these meshes the nominal mesh spacing is thus $\Delta y \cong 1 / 25=0.04$ or $\Delta y \cong 1 / 50=0.02$, while the base time-step was $\Delta t=0.01$ in most runs but half of that value was employed when deemed necessary for convergence. With view of obtaining numerical solutions that are as accurate as possible, all simulations were based on the three-time level temporal discretization (2nd order in $\Delta t$ ) and the convective fluxes in the problem of Section 4.3 were discretized with the CUBISTA scheme [36] (also formally of 2nd order accuracy).

The next sections describe the results for the following problems, tackled in order of increased complexity: Section 4.1 deals with start-up of simple channel flow; Section 4.2 deals with startup of square cross-section duct flow; Section 4.3 deals with startup of rotating duct flow.

\subsection{Start-up of simple channel flow}

Start-up flow in a channel corresponds to the transient regime resulting from application of a constant pressure gradient $P \equiv$ $-d p / d x$ to a fluid initially at rest, with the additional assumption that there are no variations of flow properties along the channel (fully developed flow in space). It is a time varying, nonhomogeneous shear flow for which there are analytical solutions in the case of Newtonian [37] and Oldroyd-B [32] fluids, thus serving as a relatively simple test problem for time-dependent viscoelastic flow assessment considered by many authors [14,16,19,20,28-31], or as a way of imposing boundary conditions at inlet [38]. After a certain interval of time the flow becomes fully established and attains a steady state. For a constant-viscosity fluid we have then a Poiseuille flow with a parabolic velocity profile

$u(y)=1.5 U\left[1-(y / H)^{2}\right]$

and the average velocity $U$, which determines the flow rate $Q=$ $2 H U$ (the channel width is $2 H$ ), is related to the pressure gradient by:

$U=P H^{2} / 3 \mu$.

This solution is also valid for the FENE-MCR and FENE-CR models, with the constant viscosity denoted by $\eta_{0}=\eta_{p}+\eta_{s}$ instead of $\mu$. For the shear-thinning FENE-P model the solution at steady state is more involved but is available [39] thus enabling a check of the code in this limiting situation of large time instants. The average velocity for the Newtonian fluid given by Eq. (25) was chosen as the normalising velocity scale in the following paragraphs; with this equation, $P=3$ gives $U=1$.

Fig. 3 shows the time evolution of velocity at the central point of the channel $(y=0)$ for the models FENE-MCR, FENE-CR and 


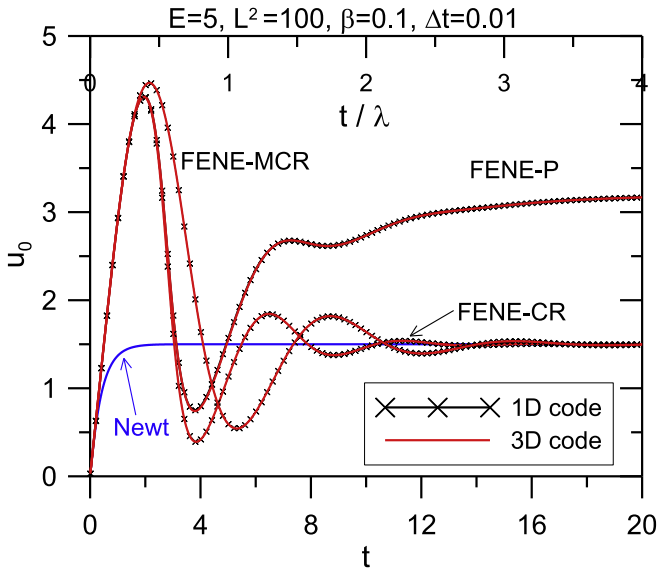

Fig. 3. Time evolution of velocity at the channel centre for the models FENE-MCR, FENE-CR and FENEP: comparison of results from the 3D simulation code and the simplified 1D program. The Newtonian result is also included for comparison.

FENE-P, and compares predictions of the simpler 1D code explained in Section 3 with those of the general multidimensional code, here denoted 3D code, using method 2. The fact that the results of the two codes are visually coincident serves as an indirect demonstration that method 2 for the FENE-CR and FENE-P models was correctly implemented in the multidimensional computer program. We emphasize that the $1 \mathrm{D}$ codes were written as an extension of previous codes used for the UCM and Oldroyd-B models [31] where exact agreement with the theory was demonstrated; they are sufficiently simple codes so that their correctness may be easily controllable (these $1 \mathrm{D}$ codes are available from the author on request). We recall that the time shown in the abscissa of Fig. 3 is normalized with a diffusion time scale and so, for $E=5$, the elapsed time in relaxation time units should be divided by 5 , as shown in the upper $x$-axis. At time instant $t=10(t=2 \lambda)$, the transient regime for the viscoleastic fluids is still evolving, but at $t=20(t=4 \lambda)$ the FENE-CR and FENE-MCR have practically attained steady state since, after a couple of initial oscillations, the central velocity is very close to 1.5 (the theoretical value in fully developed Poiseuille flow). The Newtonian fluid, shown for comparison, attains steady state much faster (at about $t \approx 2$ ); a study on the development time required by UCM and Oldroyd-B fluids, and its dependence on $E$ and $\beta$, was presented by [40] (for $E=5$ and $\beta=0.1$, it gives a start-up time of $t_{S}=27.2$, somewhat above the largest time shown in the figure), but a similar study is missing for the FENE-P and the influence of $L^{2}$.

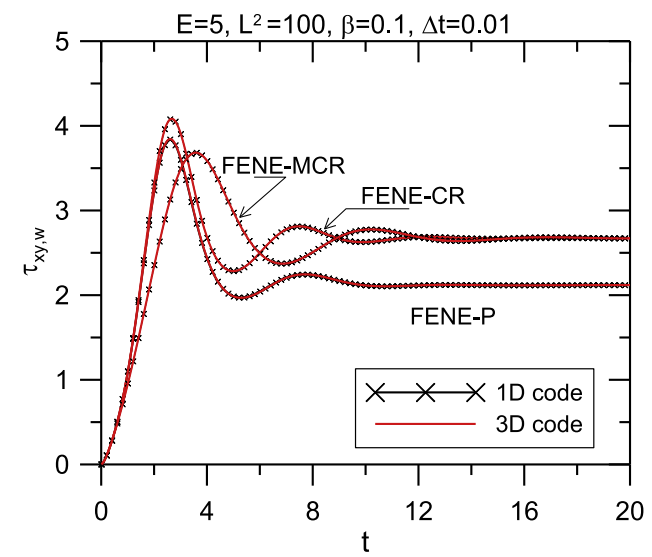

Both the shear and the main normal stresses are zero at the central line and so the stress evolution is shown at a location near the wall (nodal point of the first cell adjacent to the wall, index $w$, that is $y=0.99$ for the mesh with 101 cells here employed). Fig. 4 compares the predicted stress variation obtained with the 1D code and the main code, showing perfect agreement between results. These predictions were obtained with method 2 but, as will be shown in the next sub-section, there are virtually no differences between results of either method. After a couple of initial oscillations, the polymeric stress components for the two constantviscosity models should tend to their steady-state analytical values at the wall: $\tau_{x y, w}=3(1-\beta)=2.7$ and $\tau_{x x, w}=18 D e(1-\beta) / g$ $=29.7\left(\right.$ with $g=\left(L^{2}+\left[L^{4}+8\left(L^{2}-3\right)(3 D e)^{2}\right]^{1 / 2}\right) / 2\left(L^{2}-3\right)=2.730$ [41]), very close to those predicted at $t=20$, as seen in the figure.

In the case of the FENE-P model, Figs. 3 and 4 show that the central velocity and the near wall stresses start deviating considerably from the FENE-CR evolution at about $t \approx 4$. Since the FENE$P$ is a shear-thinning model, the application of the same pressure gradient $P=3$ results in a larger flow rate, as seen in Fig. 3 with $u_{0}$ tending to a value above 3 (when the parabolic profile has $u_{0}=1.5$ ). The actual average velocity evaluated from the predicted profile when $t>20$ and steady state is reached is $U=2.326$ and so the effective Deborah number corresponding to these FENE-P results is $D e=\lambda U / H=5 \times 2.326=11.6$.

We turn now to the main issue of the work, which is the superior performance of the new method in terms of numerical efficiency. Fig. 5 shows on the left plot the evolution of the number of iterations per time step $\left(n_{i t}\right)$ along the elapsed time, and on the right plot the total number of accumulated iterations $\left(N_{i t,}\right.$ tot $)$. The curves (in black) with symbols correspond to the standard method (method 1) and the curves (in red) without symbols to the new method (method 2), where the reduced-stress is the main dependent variable in the constitutive equations, with results shown for the FENE-P and FENE-CR models. While the overall computational work to advance the solution from $t=0$ to $t=20$ is more directly seen from plot (b) representing the total number of iterations, from which we see that the FENE-CR with the old method requires about 4 times more work than with the proposed method, and the FENE-P about 5 times more, such conclusions were already apparent from plot (a). Method 2 requires approximately a constant number of iterations per time step (of about 4-5), for both the FENE-CR and the FENE-P, along the entire time interval here simulated. Method 1 on the other hand requires a large number of iterations per time step (25-30) during the initial stages of the time evolution, when elasticity tends to promote oscillations of velocity and stresses as the flow is being established. With the old method,

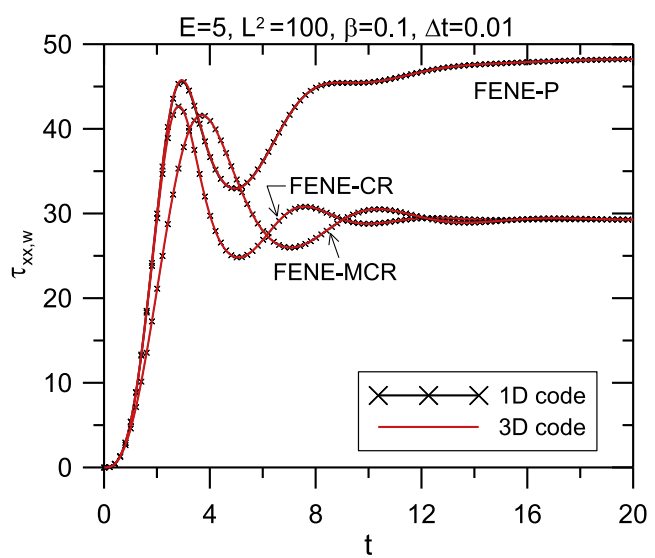

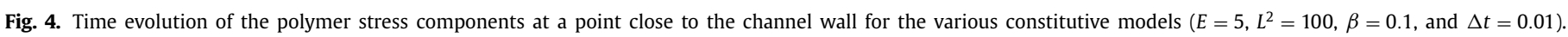
Comparison of results obtained with the 3D simulation code and the simplified 1D code. 


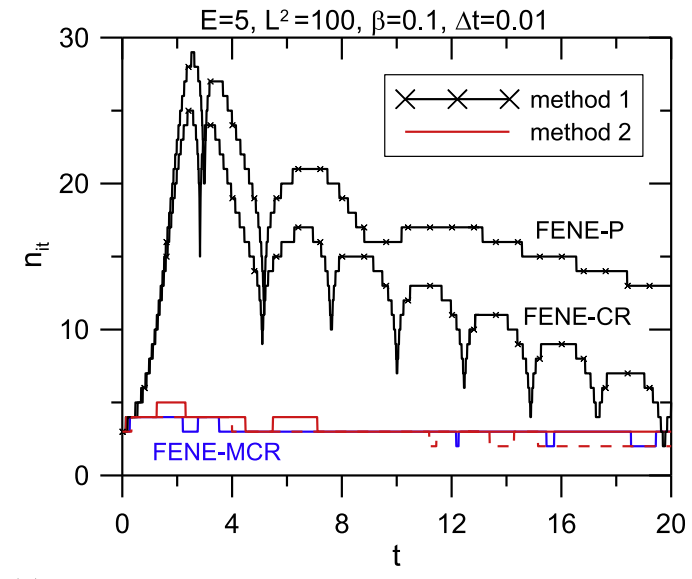

(a)

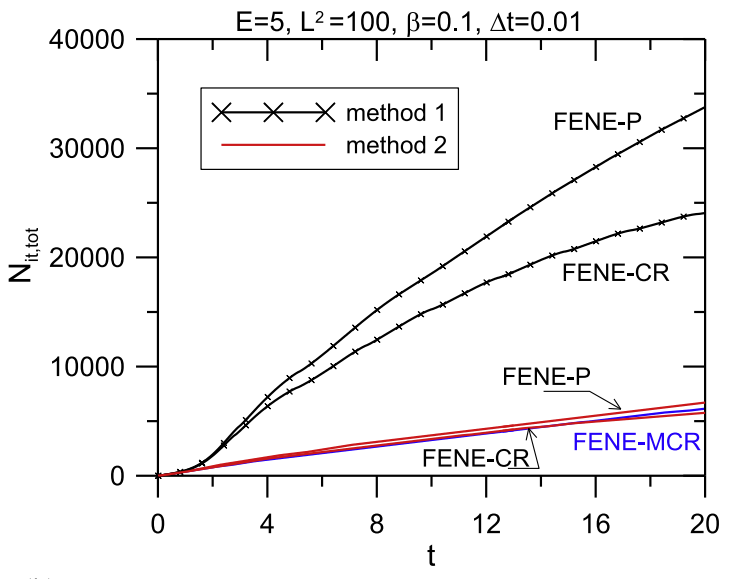

(b)

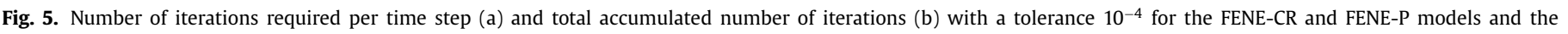
two solution methods (new method: lower curves). Results for the FENE-MCR are also shown for comparison.

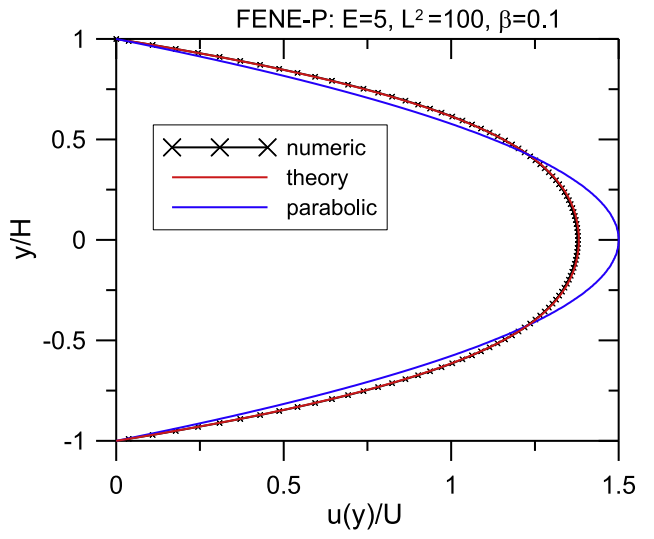

Fig. 6. Velocity profile for the FENE-P model, with $E=5$ and $P=3$, in fullyestablished regime: comparison of numerical predictions (at time $t=40$ ) and theoretical results. The parabolic profile is shown for comparison. $U$ is here the FENE-P average velocity.

the FENE-P is seen to require more iterations per time step than the FENE-CR, which is expected since the FENE-P equation is more complicate and thus introduces further inter-linkages amongst the equations for the various stress components. This figure also shows results for the FENE-MCR (the method is irrelevant), highlighting the fact that method 2 applied to FENE-P or FENE-CR behaves almost as the MCR with the standard method.

Relevant values for the iteration counts required by the two methods are given in Table 1 . We recall that the following parameters were fixed: $\beta=0.1, L^{2}=100, E=5$ (which corresponds to $D e=5$ if $U=1$ ), mesh $N Y=101$ (internal cells), $\Delta t=0.01$ and time interval $t=0-20$. Method 2 is systematically faster than method 1.

We close this section by providing a more direct verification of the predictions. This is done by comparing the numerical results under fully established conditions (at large times $t$ ), when a steady state regime has been reached, and the analytical solutions available. Since the solution (velocity and shear stress profiles) for the FENE-CR model is identical to that of the Newtonian fluid, it is more interesting to look to FENE-P results where shear-thinning immediately distorts the profiles. Fig. 6 shows velocity profiles predicted with the general simulation code with method 2 (there is no effect of the method used) at a time instant $t=40$, and the steady-state analytical solution of Cruz et al. [39] for the FENE-P. These results are virtually indistinguishable, and deviate consider- ably from the parabolic Poiseuille solution, also shown by the blue line.

Profiles of the stress components compare also quite well against the analytical solution, as shown in Fig. 7 for the same conditions. Stresses are normalized with $\eta_{0} U / H$ where $U$ is here the average velocity of the FENE-P fluid. The shear stress profile deviates from the rectilinear shape, valid for the Newtonian and FENECR fluids, on account of shear thinning, while the normal stress has an approximate quadratic variation with large values near the walls, on account of normal-stress viscoelasticity.

\subsection{Start-up of square cross-section flow}

In this sub-section we consider start-up of flow in a duct of square cross-section. Other aspect ratios could be envisaged but for the present purpose the square shape is deemed adequate. The central axis of the duct is placed at the origin of the coordinate system, $y=z=0$, and the lateral and top/bottom walls are at $y= \pm H$ and $z= \pm H$, with the square half-side $H=1$ in terms of non-dimensional quantities. Two uniform meshes were deployed on this square domain, one with $51 \times 51$ cells (nominal mesh spacing $\Delta y=\Delta z \approx 0.04$ ) and the other with $101 \times 101$ cells ( $\Delta y=\Delta z \approx 0.02$ ). As commented above, the choice of forming the mesh with an odd number of cells has the consequence that a nodal point is placed exactly at the geometrical centre of the domain, which is useful to see how the results vary with mesh refinement without the need of doing interpolations between the various meshes. For large times the flow tends to steady state and, if the fluid has a constant shear viscosity (as the FENE-CR model), the velocity field is then identical to that of the Newtonian fluid, with a known analytical solution for a $2 a \times 2 b$ rectangular crosssection (see e.g. White [33] p. 120):

$U=\frac{P a^{2}}{3 \mu}\left[1-\frac{192 a}{b} \sum_{j=1,3, \ldots}^{\infty} \frac{1}{(j \pi)^{5}} \tanh \left(\frac{j \pi b}{2 a}\right)\right]$

In order to have then a unit average velocity $U=1$ in the square cross-section $(b / a=1, a \equiv H=1)$, the pressure gradient needs to be $P \equiv-d p / d x=7.1135$, and the central velocity is $u_{0}=$ 2.096. We have decided to compare results for the same pressure gradient, and so we employ here, for all simulations, $P=7.1135$, together with $E=5$ (that is $\lambda=5$ ), $L^{2}=100, \beta=0.1$ (that is $\eta_{s}=$ $0.1, \eta_{p}=0.1$ and $\eta_{0}=1$ ). This means that for the FENE-P fluid the average velocity at steady state will be larger than unity; the simulations give $U=2.92$ (there is no analytical solution). 

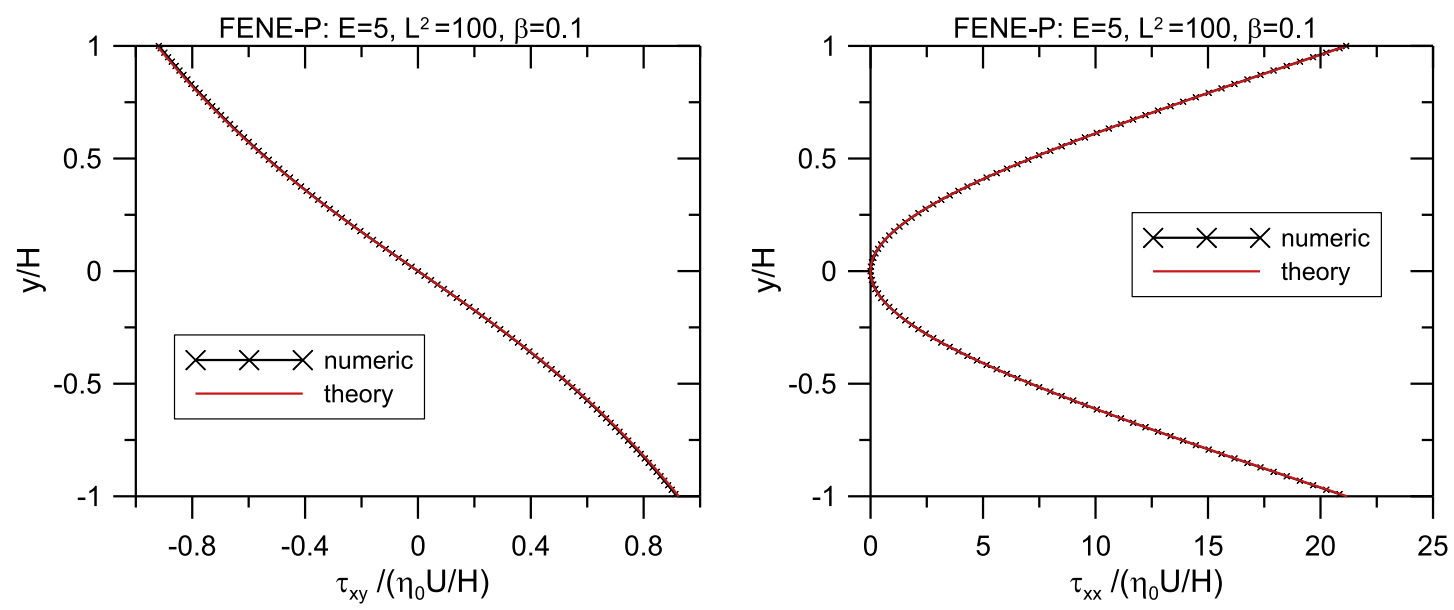

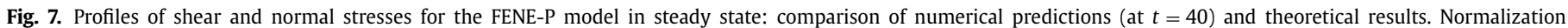
with the FENE-P average velocity $U$.
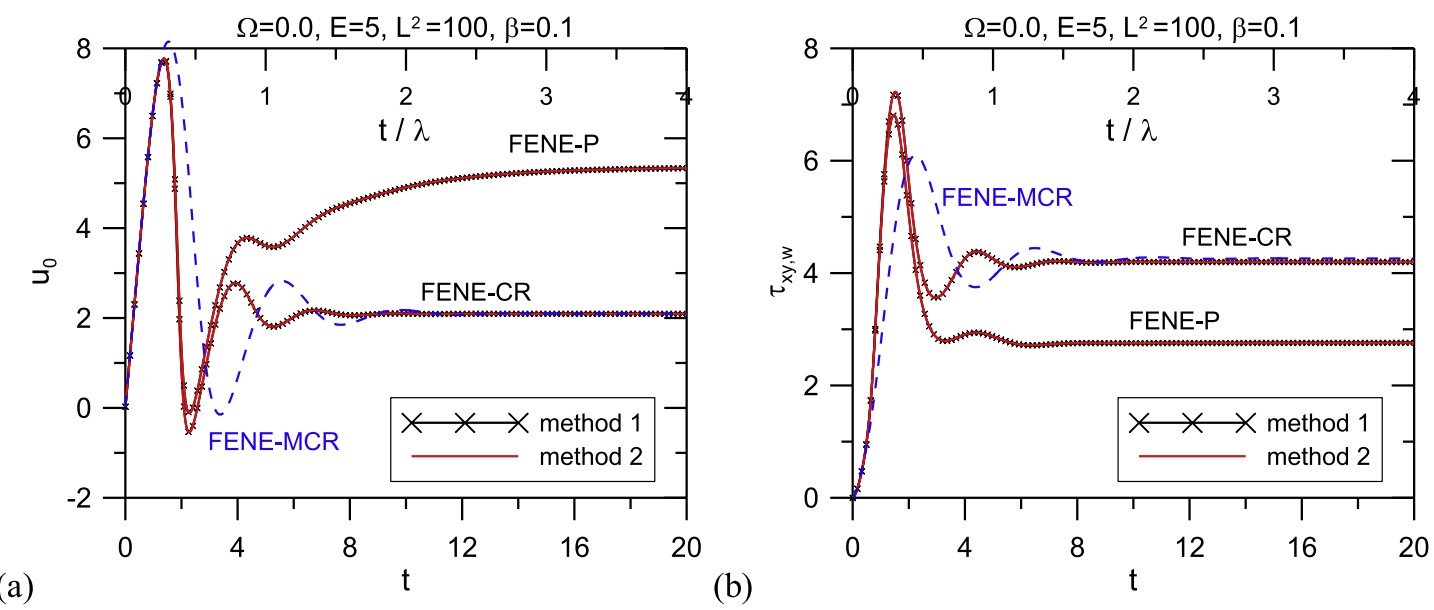

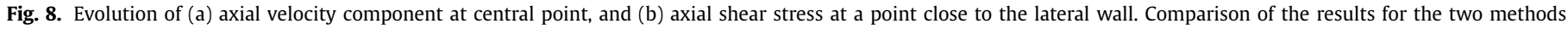
with three constitutive models (Mesh $101 \times 101$; time step $\Delta t=0.004$ ).

First, we demonstrate that the two methods used to solve numerically this problem yield the same physical results. Fig. 8 show the $x$-velocity component at the central point of the cross-section $\left(u_{0}\right)$ and the $x y$-component of the shear stress at a point adjacent to the lateral wall $\left(\tau_{x y, w}=\tau_{x y}(y=0 ; z=-0.990)\right)$, during the transient regime corresponding to the start-up of the flow. These predictions were obtained on the finer mesh, with $101 \times 101$ cells. We let time evolve from zero to 20 (recall, diffusive time scale $\left.H^{2} /\left(\eta_{0} / \rho\right)\right)$ which seems sufficient for the flow to attain a steady state, after a few oscillations typical of viscoelastic flow with a non-zero solvent viscosity $[14,16,31,32,38]$. After the first oscillation the FENE-P model follows a different path of evolution compared to the constant viscosity models, and takes longer to attain a steady regime. At large times, the central velocity for the FENEP tends to 5.34 , or $u_{0} / U=1.83$ if made non-dimensional with the FENE-P average velocity $(U=2.917)$, being thus smaller than that for the constant-viscosity fluids, an effect due to shear-thinning. Initially all models follow the same curve, but afterwards the amplitude and frequency of the response differ. The main point for the present purposes is that the two methods, the standard stress method (method 1) and the reduced stress method (method 2), predict the same numerical solution (coincidence of the curves in the figure), thus reinforcing confidence in the correct implementation of the new method. The FENE-MCR solution is shown for the purpose of comparison against the FENE-CR; it was obtained with method 1.

The distribution of the number of iterations per time step during the simulation using the two methods is shown in Fig. 9, for the FENE-P (Fig. 9a) and FENE-CR (Fig. 9b), and comparing the requirements of two meshes $51 \times 51$ and $101 \times 101$. Again, the reduced stress method (method 2 ) requires a much smaller number of iterations, tending to about 3-4 iterations at each $\Delta t$ as the steady state is approached and a somewhat larger number in the initial part of the simulation when the strong dynamical oscillations due to viscoelasticity are felt. The iteration numbers are insensitive to the mesh fineness. On the other hand, the method previously employed uses many iterations per time step (raising to about 50-60, in the initial stages, for the convergence tolerance of $10^{-4}$ here employed), these tend to rise appreciably when the mesh is refined, and things are worst for the more complex FENE$P$ model, while the FENE-CR shows a more marked decay of $n_{i t}$ as the flow evolves to the steady state (which should be attained not too later than $t=20$ ). It is interesting to observe from Fig. 9b the wave-like evolution of the number of iterations per time step for the FENE-CR which is the result of the propagation of the stress discontinuities created when the flow is initiated (near each channel wall), travelling from wall to wall with a wave speed proportional to $\sqrt{(1-\beta) / E}$ and interfering at the central line every 2.36 

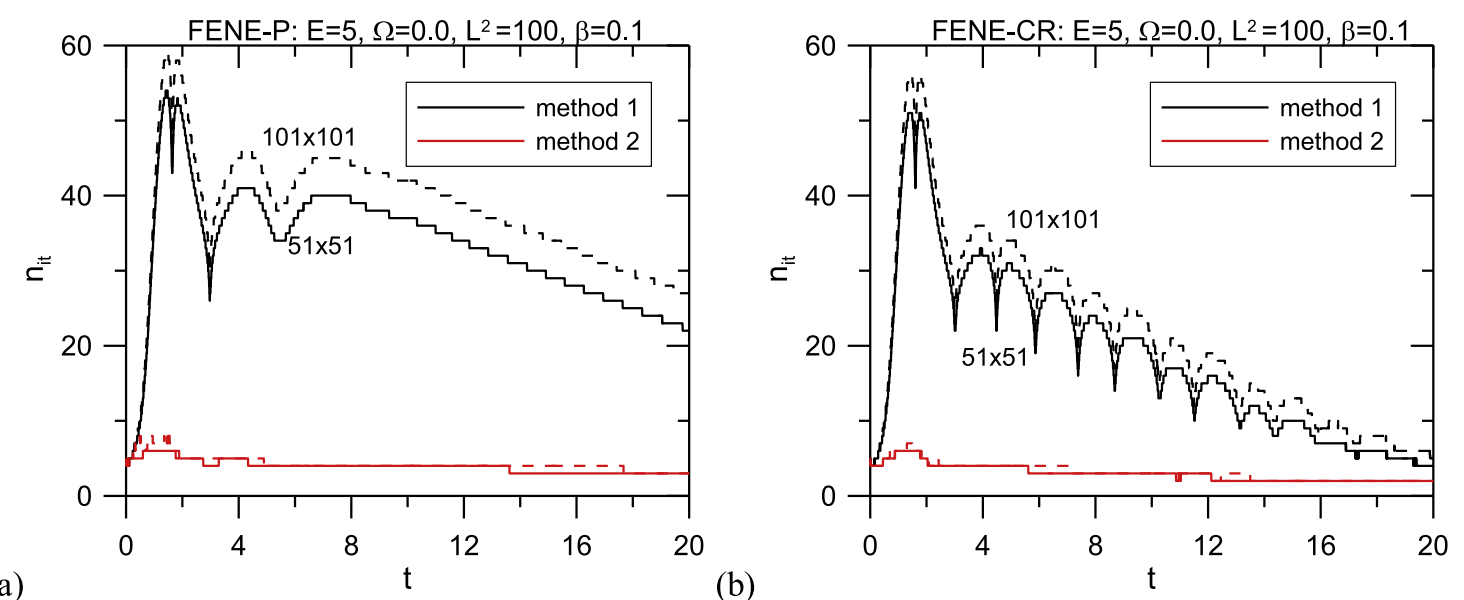

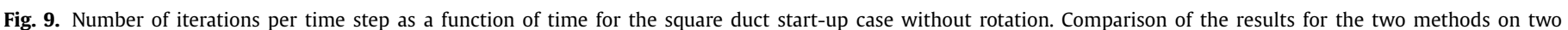
grids $(51 \times 51$ and $101 \times 101)$ : (a) FENE-P; (b) FENE-CR.

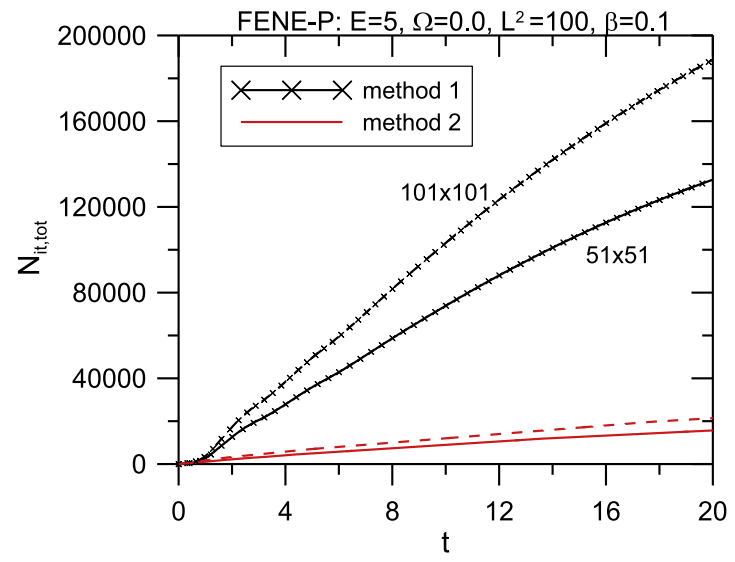

(a)

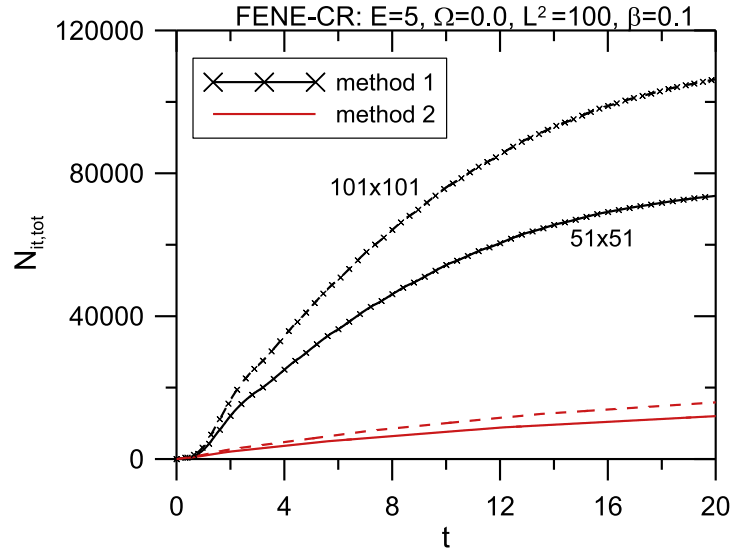

(b)

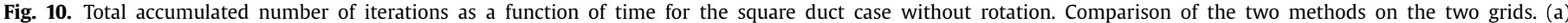
FENE-P; (b) FENE-CR.

time intervals (see Eq. (27) in [39] for Oldroyd-B fluid, taken as an approximation to the FENE-CR).

Computational work and speed of calculations should be proportional to the total number of iterations $N_{i t, \text { tot }}$ and these are shown as a function of simulation time in Fig. 10a for the FENE$\mathrm{P}$ model and Fig. 10b for the FENE-CR, where results obtained with the two methods on two meshes are compared. Method 1 requires a much smaller number of total iterations compared to method 2 (about 8 times less on mesh $51 \times 51$, and 9 times less on mesh $101 \times 101)$. In addition, method 2 is less affected by the degree of mesh refinement, the figure showing just a marginal increase of $N_{i t, \text { tot }}$ when the number of grid points is quadruplicated. Relevant data for computational cost assessment in this test problem is provided in Table 2. These data for the FENE-P model on the fine mesh show a speed-up of 9.4 fold in terms of CPU time (which should depend on the machine employed) and 8.8 fold in terms of total number of iterations (independent of the machine), at simulation time $t=20$. The average number of outer iterations in each time step raises from about 4 with the reduced-stress method to 30 with the standard stress method. For the FENE-CR model the corresponding ratios are 7.1 (CPU time) and 6.7 (total iterations).

\subsection{Start-up of rotating duct flow}

Here the duct is the same of the previous subsection, but now it rotates about the vertical $z$-axis with speed $\Omega$. The flow is com- puted in the non-inertial frame fixed to the rotating duct (same $u, v, w$ velocity components as before) and so the relative movement gives rise to a pseudo Coriolis force $\mathbf{F}_{C}=-\rho 2 \boldsymbol{\Omega} \times \mathbf{v}$ with components:

$F_{C, x}=2 \rho \Omega v, F_{C, y}=-2 \rho \Omega u, F_{C, z}=0$

which need to be added to the right-hand side of the momentum equations (Eq. (1)). We assume $\boldsymbol{\Omega}=\Omega \hat{\mathbf{z}}$, where $\Omega$ is the angular velocity component about the $z$-axis. In non-dimensional terms, $\Omega$ is the inverse of the Ekman number, usually defined as $E k=\left(\eta_{0} / \rho\right) / \Omega H^{2}$, with, here, $\eta_{0}=1, \rho=1$ and $H=1$. So, $\Omega>1$ implies preponderance of Coriolis rotational effects, since the time scale related to frame rotation becomes smaller than the flow diffusion time and $E k<1$. We have tried various values of rotation speed, from 0 to 10 , but the results to be presented below are for $\Omega=2$. Note that the main effect of the Coriolis force results from its $y$-component, pushing fluid towards the left of the main flow direction (i.e. in the minus $y$-direction) along the horizontal central plane $(z=0)$ and thus creating a secondary flow, in the $y$ $z$ plane, composed by two counter-rotating recirculating cells (cf. Fig. 1). The $x$-component of the Coriolis force also acts to distort the main (axial, along $x$ ) velocity profile but since $v \ll u$ its effect is much smaller than that of $F_{C, y}$. Fig. 11 illustrates the secondary flow pattern by means of a velocity vector plot and the corresponding streamlines for a Newtonian fluid. In this case, the average velocity is found to be slightly below unity, $U=0.992$, and 
Table 2

Computational cost for the non-rotating duct case $(\Omega=0)$ : number of iterations and computer times for the various constitutive models and solution methods.

\begin{tabular}{|c|c|c|c|c|c|}
\hline & \multirow{2}{*}{$\begin{array}{l}\text { FENE-MCR } \\
\text { Method } 1\end{array}$} & \multicolumn{2}{|l|}{ FENE-CR } & \multicolumn{2}{|l|}{ FENE-P } \\
\hline & & Method 1 & Method 2 & Method 1 & Method 2 \\
\hline \multicolumn{6}{|l|}{ (a) Mesh $51 \times 51$} \\
\hline Total number iter. & 13,163 & 73,686 & 12,018 & 132,700 & 15,689 \\
\hline Average $\mathrm{n}^{\circ}$ iter. & 3.29 & 18.42 & 3.00 & 33.18 & 3.92 \\
\hline Max. iter. per $\Delta t$ & 5 & 51 & 6 & 54 & 6 \\
\hline Iter. per $\Delta t$ at end & 2 & 4 & 2 & 22 & 3 \\
\hline CPU time $(\mathrm{s})$ & 170 & 869 & 148 & 1740 & 206 \\
\hline \multicolumn{6}{|l|}{ (b) Mesh $101 \times 101$} \\
\hline Total number iter. & 17,811 & 106,322 & 15,871 & 188,716 & 21,432 \\
\hline Average $\mathrm{n}^{\circ}$ iter. & 3.56 & 21.26 & 3.17 & 37.74 & 4.29 \\
\hline Max. iter. per $\Delta t$ & 5 & 56 & 7 & 59 & 8 \\
\hline Iiter. per $\Delta t$ at end & 3 & 5 & 2 & 27 & 3 \\
\hline CPU time $(\mathrm{s})$ & 768 & 4754 & 666 & 11,445 & 1224 \\
\hline
\end{tabular}
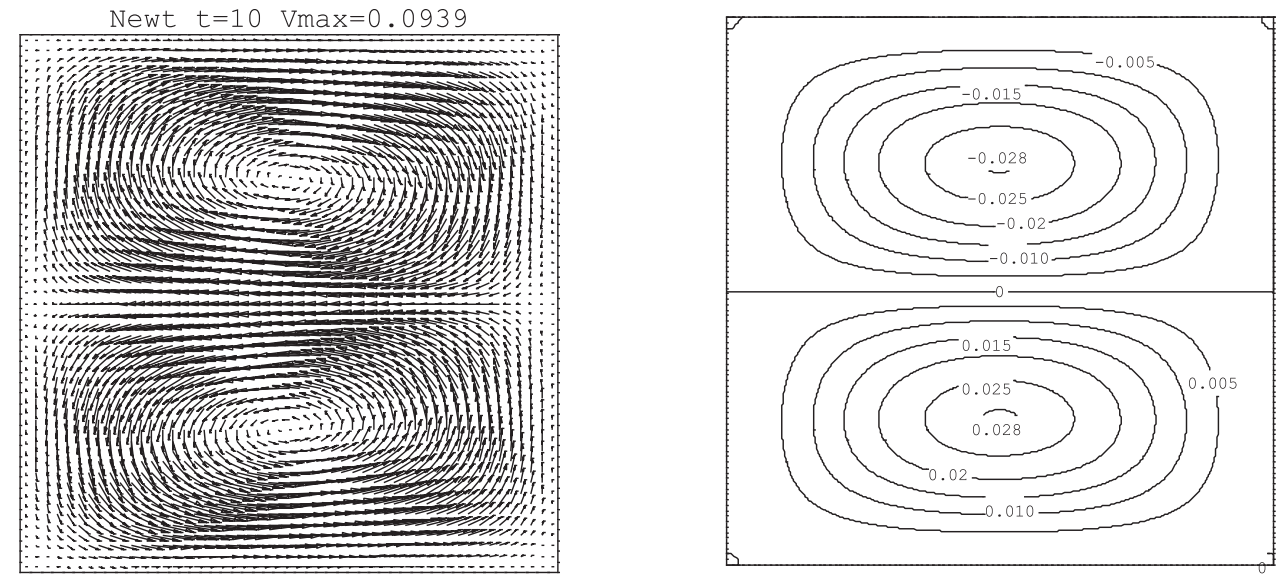

(a)

(b)

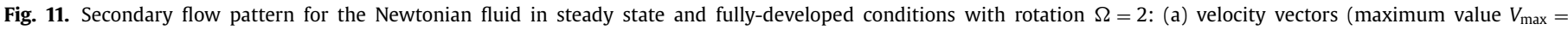
0.0939); (b) Streamlines (maximum value $\psi_{\max }=0.0282$ ).
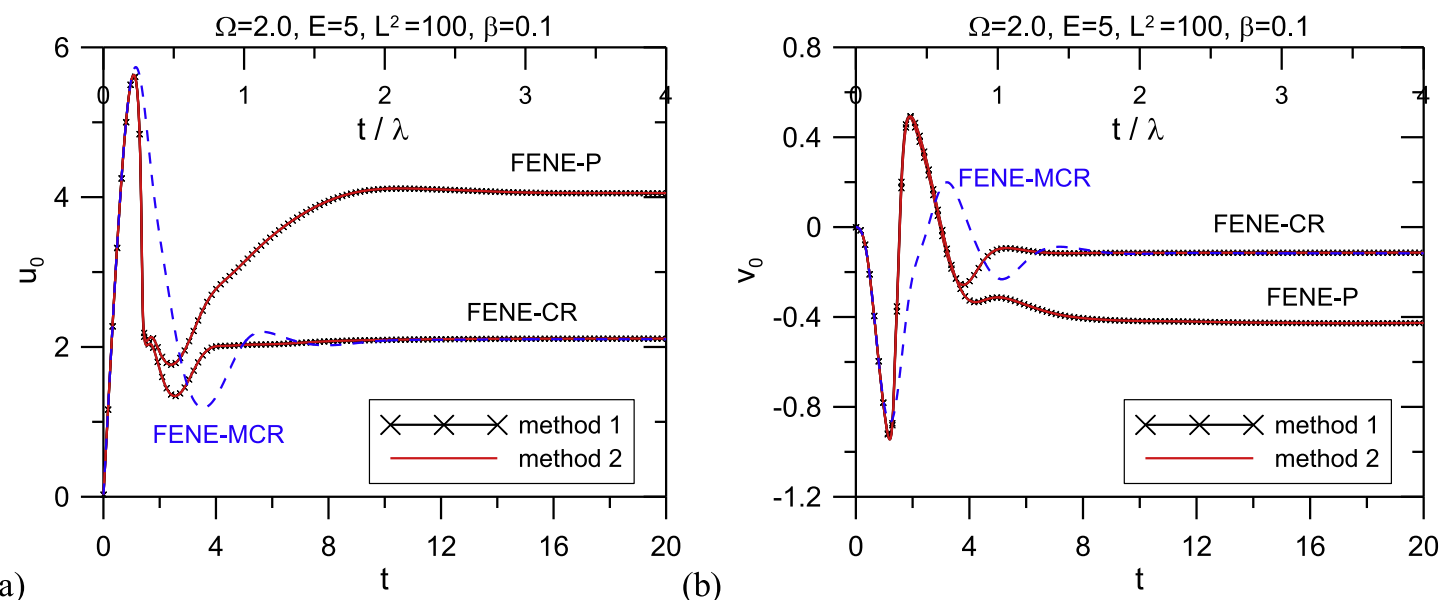

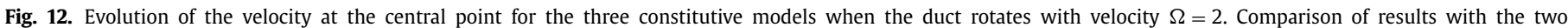
methods (Mesh $101 \times 101$; time step $\Delta t=0.004$ ): (a) axial $x$-component; (b) lateral $y$-component.

the axial velocity at the central point $u_{0}=2.060$ is also somewhat lower than the analytical result for the non-rotating case (2.096). The magnitude of the larger velocity vector in the cross-section plane is $9.4 \%$ of the main average velocity and the intensity of the flow in recirculation is $2.8 \%$ of the axial flow rate.

For the viscoelastic fluids, the evolution of velocity at the central point is shown in Fig. 12 to be compared with Fig. 8 for the non-rotating case. The two methods give the same predictions of all quantities in this complex flow problem, where all three velocity components, pressure, and the six stress components need be computed, once more demonstrating the correct implementation of method 2 (which in fact is relatively easy to do in an existing code). Regarding physical aspects of the problem, we see that the velocity fields (and also the stresses, not shown for brevity) 

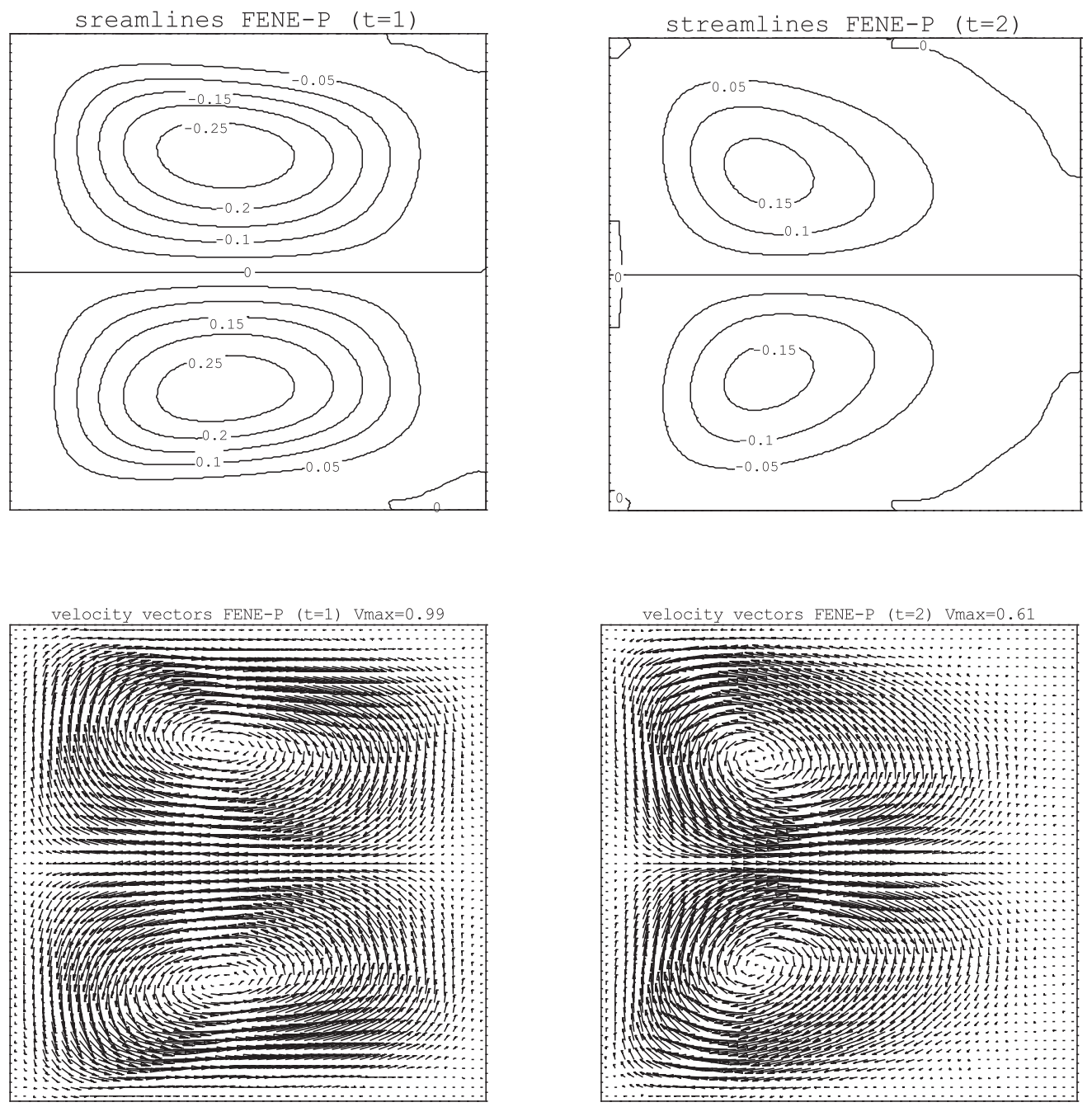

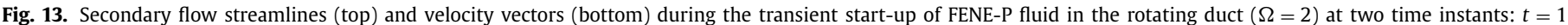
(left column; upper cell rotates clockwise, streamfunction $\psi<0$ ) and $t=2$ (right column; upper cell rotates anti-clockwise, $\psi>0)$. Note $V_{\text {max }}=M a x\left(\sqrt{v^{2}+w^{2}}\right)$.

evolve differently for the three models, except in the initial times (up to about half a relaxation time) when FENE-P and FENE-CR follow similar paths, but FENE-MCR is already deviating (the initial oscillation has a longer period and less sharp variations). It is interesting to notice in Fig. 12b that the lateral velocity is negative at large times, when the typical Coriolis cell becomes fully established, with fluid going along the $-y$ direction at the central plane $(z=0)$, but for these viscoelastic fluids a kind of recoil phenomenon occurs during the transient regime: $v_{0}$ is initially negative, due to the influence of the Coriolis force $F_{C, y}$, but at $t=2$ (about half a relaxation time) the elastic nature of the fluid pulls the fluid elements back to their original configuration, even overshooting the initial position which is seen by the positive value of the $v$-velocity component. This means that the recirculation cells turn now in a direction opposite to that determined by the Coriolis force.

Such recoil phenomena, typical of the time-dependent flow of viscoelastic liquids, is illustrated in Fig. 13 by means of streamlines of the secondary flow in the cross-section plane $(y-z)$ and the corresponding velocity vectors field, for the FENE-P fluid model. At time $t=1$ after inception of the flow (with the axial pressure gradient $P=-d p / d x=7.1135$ and the angular rotation velocity $\Omega=2$ applied suddenly at $t=0$ to the fluid initially at rest) the previous figure (Fig. 12) suggests that the flow is still in the initial accelerating regime, with preponderance of Coriolis force generating the distinctive recirculating cell pair: fluid is pushed to the left (minus $y$-direction; in the figure the $y$-axis is to the right, the $z$-axis to the top, and the $x$-axis leaves the page towards the viewer) and so the upper cell has clockwise rotation (negative stream-function values $\psi$, with $w=-\partial \psi / \partial y$ and $v=\partial \psi / \partial z$ ) and the lower cell has anti-clockwise rotation. However, at $t=2$ the reversed situation is observed: the upper cell rotates anti-clockwise (positive $\psi$ ) and the lower cell clockwise. The centres of the re-circulations are also seen to be shifted to the left. Physically the situation can be explained as follows: polymer molecules are initially stretched as Coriolis force deviates the fluid to the left and thus creates a elongational flow along the central plane $y=0$; the molecules then relax, after a certain time delay, and generate a flow along the opposite direction - that is, the recoil phase. This situation was seen as the single large velocity oscillation in Fig. 12 eventually giving $v_{0}>0$; viscous effects will then tend to dump the elastic recoil effect, and at steady state the flow has the typical pair of cells related to overall duct rotation. Recoil is often found in viscoelastic liquid systems; Pakdel et al. [42] reports a similar recoil effect observed in experiments during the transient set up of a lid-driven cavity flow.

The variation of the number of iterations per time step is shown in Fig. 14 for the FENE-P and FENE-CR models on the two meshes. It is clear that method 2 requires much less iterations on average, by a factor of about 7 , with representative values of 

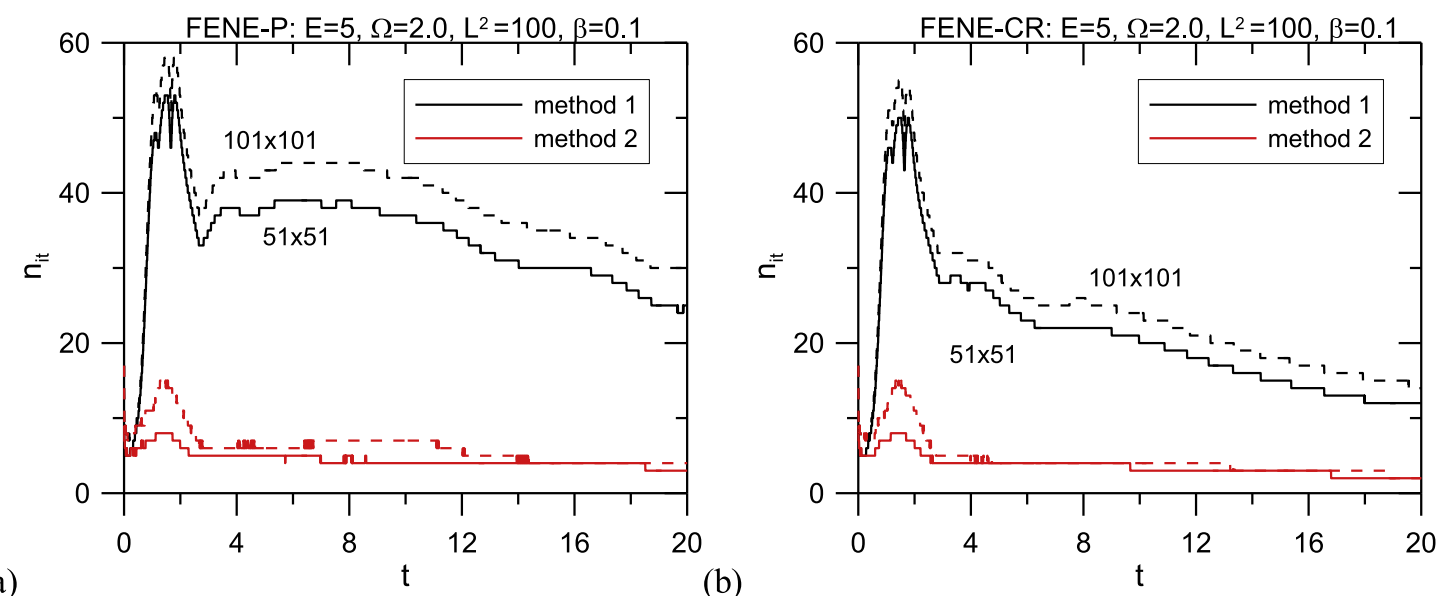

(a)

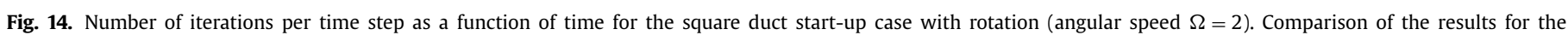
two methods on two grids $(51 \times 51$ and $101 \times 101)$ : (a) FENE-P; (b) FENE-CR.
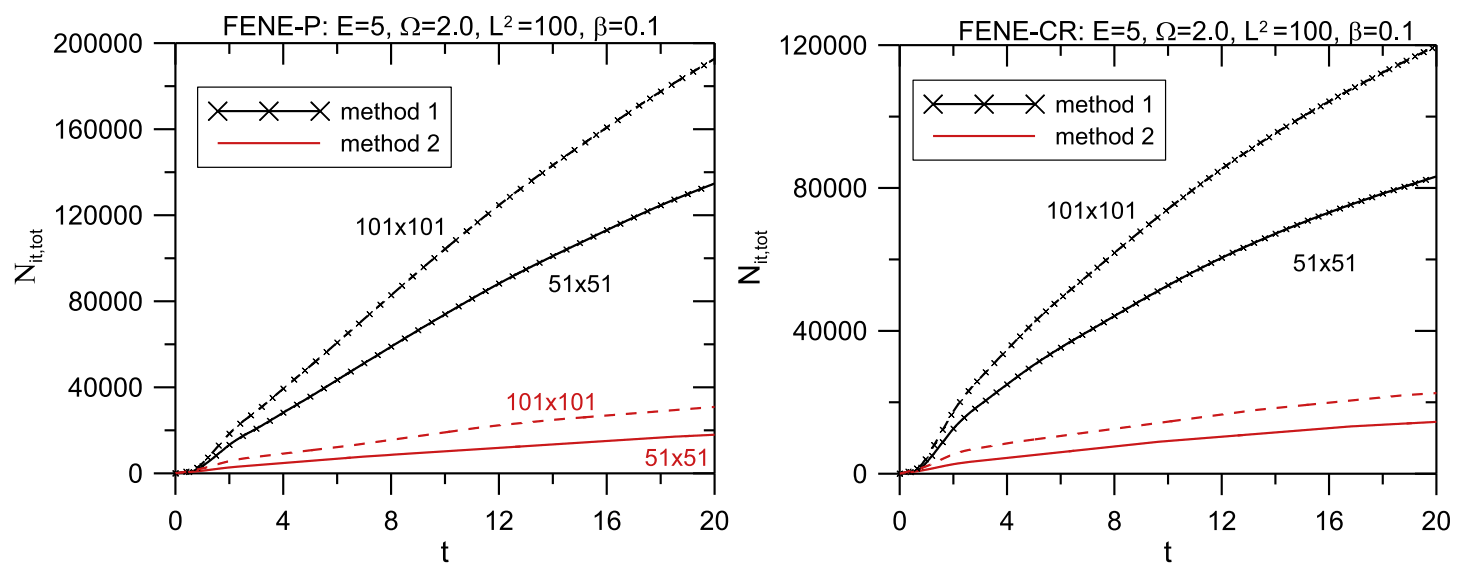

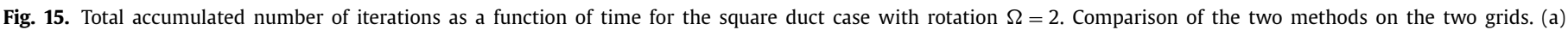
FENE-P; (b) FENE-CR.

about 6 iterations for the FENE-P and 4 for FENE-CR. The standard method requires about 40 iterations per $\Delta t$ for the FENE-P model and around 25 to 30 iterations with the FENE-CR, values that only start decreasing as the steady state is approached. Higher numbers of iterations per time step are generally required in the initial part of the simulation, for times from $t=1$ to $t=2$, when the physical oscillation due to viscoelasticity takes place and the dependent variables change more abruptly.

The computational cost is more directly related to the total number of iterations taken up to a given time instant, obtained by summing all previous outer iteration numbers. In this way even the fact that different time steps might have been used in the various simulations is duly taken into account. Fig. 15 shows the corresponding plots, in which we emphasize again that the ordinate is proportional to the computational cost. Not only does the new method requires much less total iterations, but it is also less sensitive (on the scales shown) to mesh refinement and exhibits a lower rate of increase as time progresses. On the fine mesh, for the FENE$P$ model, the reduced stress method required 30,896 iterations at $t=20$, with a CPU time of $1797 \mathrm{~s}$ (about $30 \mathrm{~min}$ ); the standard method used 192,715 iterations and CPU of $12388 \mathrm{~s}$ (about $3 \mathrm{~h}$ and $26 \mathrm{~min}$ ). Hence, the new method entails a reduction of about 6.9 fold in computer time, while the ratio of total iterations is 6.2, showing that $N_{i t, \text { tot }}$ provides an adequate measure of the computational cost. More data illustrating the computational gains achieved be the new reduced-stress method are provided in Table 3: the total number of iterations at $t=20\left(N_{i t, t o t}\right)$; the average number of iterations $\left(\bar{n}_{i t}=N_{i t, t o t} / N_{t}\right.$ where $N_{t}$ is the number of time steps, $\left.N_{t}=t_{\text {final }} / \Delta t\right)$; the maximum number of iterations per time step $\left(n_{i t, \max }\right)$; the number of time steps at the end of the simulation, here taken as $t_{\text {final }}=20\left(n_{\text {it, final }}\right)$; the CPU time in the PC with processor Intel Core i7-5500 U at $2.40 \mathrm{GHz}$.

Finally, we provide in Table 4 some useful data for benchmarking when the frame-rotation velocity (that is, the inverse of the Ekman number) is raised from $\Omega=0$ to $\Omega=10$. We recall that in all previous simulations we used the value $\Omega=2$. It is seen that as $\Omega$ increases the average velocity $U$ is reduced, as does the streamwise velocity component at the center $u_{0}$, on account of more intense Coriolis-induced recirculation cell patterns for the same input force $P=-d p / d x$. A measure of the strength of those recirculations is given by the lateral velocity at the center $v_{0}$ (at $t=20$ ): in the case of the FENE-P, it reaches $17.5 \%$ of the average velocity for $\Omega=2$ and increases to $20.5 \%$ for $\Omega=4$, after which it tends to reduce again slightly. Such trend may be explained as an effect of shear-thinning in viscosity, since for the FENE-CR the results in Table 4 show a steady increase of the central lateral velocity $v_{0}$ as is the rotation velocity $\Omega$ is increased. Similarly, data given in Table 4 for the extrema of the stream-function related to the secondary flow $\left(\psi_{\max }\right.$ and $\psi_{\min }$, with $\psi_{\min }=-\psi_{\max }$ due to the verified symmetry of that flow) replicate the same conclusions on the influence of shear thinning; it is seen that the secondary flow is about $14 \%$ of the main flow rate for the FENE-P model and $7 \%$ for the FENE-CR. For this latter model, owing to its Boger fluid-like characteristics, $\psi_{\max }$ is always increasing with $\Omega$. The maximum 
Table 3

Computational cost for the rotating duct case $(\Omega=2)$ : number of iterations and computer times for the various constitutive models and solution methods.

\begin{tabular}{|c|c|c|c|c|c|}
\hline & \multirow{2}{*}{$\begin{array}{l}\text { FENE-MCR } \\
\text { Method } 1\end{array}$} & \multicolumn{2}{|l|}{ FENE-CR } & \multicolumn{2}{|l|}{ FENE-P } \\
\hline & & Method 1 & Method 2 & Method 1 & Method 2 \\
\hline \multicolumn{6}{|l|}{ (a) Mesh $51 \times 51$} \\
\hline Total number iter. & 15,326 & 83,245 & 14,524 & 134,762 & 17,955 \\
\hline Average $n^{\circ}$ iter. & 3.83 & 20.81 & 3.63 & 33.69 & 4.49 \\
\hline Max. iter. per $\Delta t$ & 9 & 50 & 9 & 53 & 9 \\
\hline Iter. per $\Delta t$ at end & 3 & 12 & 2 & 25 & 3 \\
\hline CPU time (s) & 226 & 1198 & 201 & 1892 & 255 \\
\hline \multicolumn{6}{|l|}{ (b) Mesh $101 \times 101$} \\
\hline Total number iter. & 22,380 & 119,746 & 22,584 & 192,715 & 30,896 \\
\hline Average $\mathrm{n}^{\circ}$ iter. & 4.48 & 23.95 & 4.52 & 38.54 & 6.18 \\
\hline Max. iter. per $\Delta t$ & 17 & 55 & 17 & 58 & 17 \\
\hline liter. per $\Delta t$ at end & 3 & 14 & 2 & 30 & 4 \\
\hline CPU time (s) & 1377 & 7526 & 1385 & 12,388 & 1797 \\
\hline
\end{tabular}

Table 4

Average velocity, velocity components at the central point and maxima secondary stream-function for the FENE-P and FENE-CR models at time $t=20$ (mesh $101 \times 101$ ). Also given are the maximum and minimum lateral velocities during the transient process and the time instants at which they occur.

\begin{tabular}{lllllllll}
\hline FENE-P & & & & & & & \\
\hline$\Omega=$ & $U$ & $u_{0} / U$ & $v_{0} / U$ & $\psi_{\max , \min }$ & $\left(v_{0}\right)_{\min } / U$ & $t$ & $\left(v_{0}\right)_{\max } / U$ & $t$ \\
\hline 0 & 2.92 & 1.83 & 0 & 0 & 0 & - & 0 & - \\
2 & 2.43 & 1.66 & -0.175 & \pm 0.119 & -0.388 & 1.195 & +0.203 & 1.915 \\
4 & 2.13 & 1.47 & -0.205 & \pm 0.140 & -0.431 & 1.185 & +0.0184 & 1.645 \\
6 & 1.97 & 1.38 & -0.202 & \pm 0.143 & -0.359 & 1.084 & -0.0474 & 2.300 \\
8 & 1.85 & 1.33 & -0.190 & \pm 0.138 & -0.297 & 1.285 & -0.0700 & 2.785 \\
10 & 1.76 & 1.30 & -0.178 & \pm 0.131 & -0.261 & 1.205 & -0.0790 & 3.270 \\
FENE-CR & & & & & & & \\
\hline$\Omega=$ & $U$ & $u_{0} / U$ & $v_{0} / U$ & $\psi_{\max , \min }$ & $\left(v_{0}\right)_{\min } / U$ & $t$ & & $\left(v_{0}\right)_{\max } / U$ \\
\hline 0 & 1.00 & 2.10 & 0 & 0 & 0 & - & 0 & $t$ \\
2 & 1.01 & 2.09 & -0.112 & \pm 0.0328 & -0.930 & 1.195 & +0.486 & 1.880 \\
4 & 0.976 & 1.88 & -0.191 & \pm 0.0562 & -0.941 & 1.185 & +0.0498 & 2.395 \\
6 & 0.927 & 1.73 & -0.227 & \pm 0.0676 & -0.762 & 1.085 & -0.103 & 3.025 \\
8 & 0.884 & 1.63 & -0.242 & \pm 0.0731 & -0.631 & 1.280 & -0.175 & 3.755 \\
10 & 0.847 & 1.55 & -0.247 & \pm 0.0753 & -0.552 & 1.205 & -0.205 & 4.520 \\
\hline
\end{tabular}

and minimum values of $v_{0}$ during the transient flow set-up period are also registered in Table 4; the minimum (negative) value, representing cell rotation induced by Coriolis effects, occurs first at an earlier time when the flow is still at an accelerating stage (no differences between FENE-P and FENE-CR models). The maximum value $\left(v_{0}\right)_{\max }$, which may be positive (cells rotating in the opposite direction, due to elastic recoil) or negative (the direction of rotation of the secondary cells is the same as that imposed by Coriolis effects, in spite of some recoil being present), appears to peak at a rotation of about $\Omega \approx 2$. Regarding mesh refinement and the uncertainty of the present numerical results, the differences between predictions (FENE-P, $\Omega=2, E=5$ ) on meshes $51 \times 51$ and $101 \times 101$ are only of about $0.1 \%$ for $U, u_{0}$ and $\left(v_{0}\right)_{\max }$, raising to about $1 \%$ for $v_{0}$ (at $t=20$ ) which is a rather small number.

\section{Conclusions}

It has been shown that the numerical solution of timedependent viscoelastic flow problems, whose fluids follow a differential constitutive equation of the FENE-P type (represented here by the FENE-CR or FENE-P models) is much more efficient when the method 2 here described is employed, in which the dependent variable is a reduced stress defined as $\tau^{\prime}{ }_{i j}=\tau_{i j} / f\left(\tau_{k k}\right)$, where $f$ is the function of the trace of the stress tensor $\tau_{k k}$ that describes the spring force in the kinetic model. Compared to the classical application of standard integration methods (such as the implicit Euler or the 3-time-level method) to the original constitutive equa- tions, the new formulation brings in decisive advantages in terms of computational speed, allowing the numerical solution for the time evolution of the stresses to be effected in a factor of 5 to 10 fold faster.

As shown in the Appendix, gains in efficiency essentially result from a numerical effect, whereby the iteration errors from the standard method (method 1 ) are related to a fixed-point constant $\left(F^{\prime} \equiv|\partial F / \partial X|\right.$, for an iteration process $X=F(X)$ where the iterated variable is $X=\tau_{x x}, \tau_{x y}$, etc.) proportional to $F^{\prime} \cong\left(\tau_{x x}^{n} / L^{2}\right) / f^{n}$, which is of order $\sim 1$ at most, although slightly smaller than unity (otherwise the fixed-point iteration would not converge, since errors at successive iterations $k$ decay as $\left.e_{k+1}=\left|F^{\prime}\right|^{k} e_{1}\right)$. In contrast, with method 2 the fixed-point constant is of order $F^{\prime} \cong \Delta t\left(\tau_{x x}^{\prime n} / L^{2}\right)$, that is $\sim 10^{-2}-10^{-1}$, and so convergence tends to occur with much less number of iterations.

A novel flow problem was used as test case, namely the startup of viscoelastic flow in a rotating square duct after sudden imposition of a pressure gradient, where rotational speed tends to generate a pair of counter-rotating recirculating secondary flow cells. Coriolis acceleration for a Newtonian fluid promotes a deviation to the left along the "horizontal" $(z=0)$ plane which gradually establishes a secondary flow, with an upper cell rotating clockwise and a lower cell rotating anti-clockwise. For the viscoelastic fluids the same occurs at larger times, when a steady flow is eventually established, but it was found that during the transient process a recoil phenomenon tends to generate a pair of cells rotating with opposite directions of those present when the steady state is reached. 


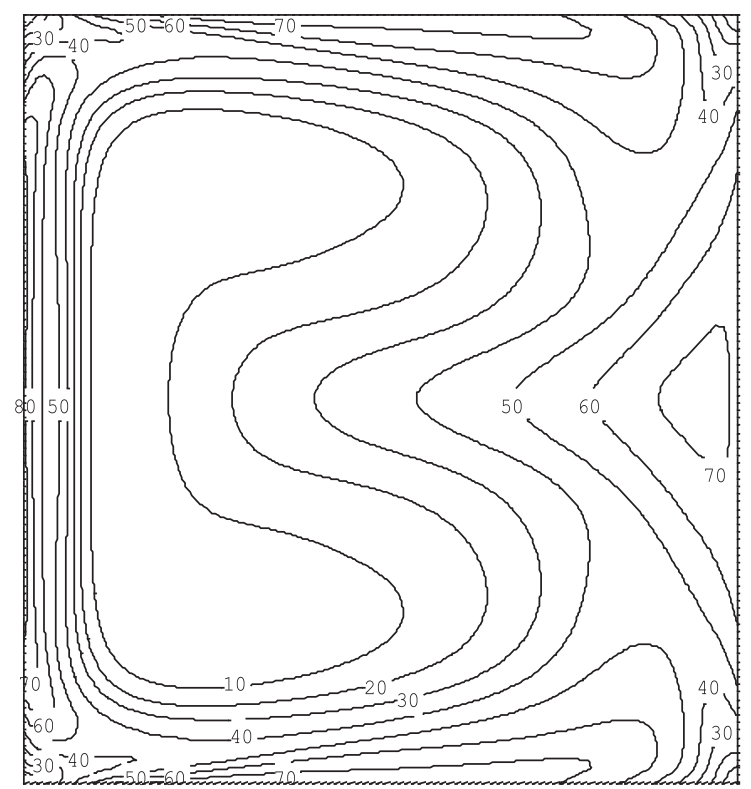

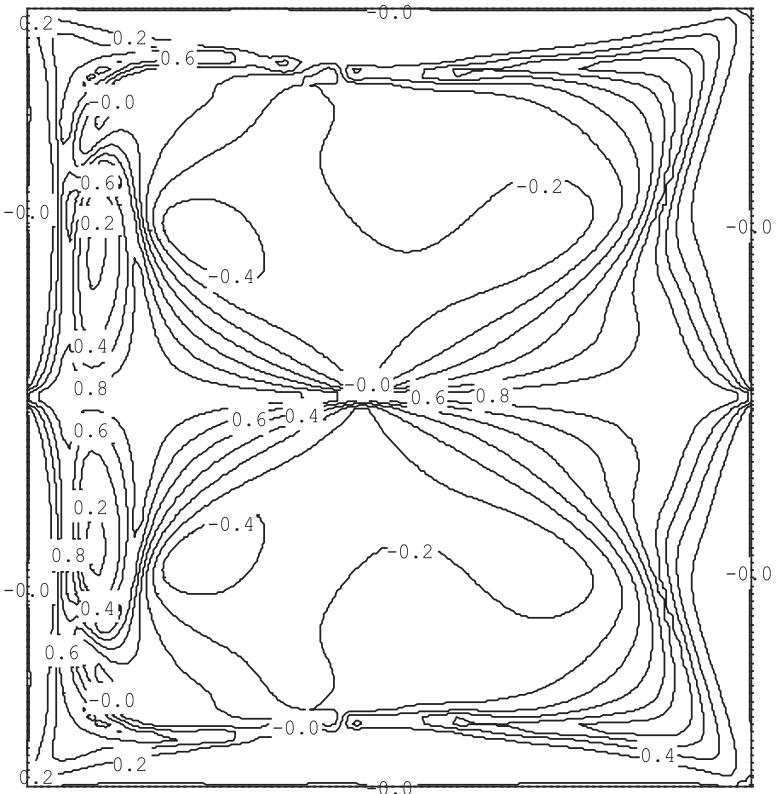

a)

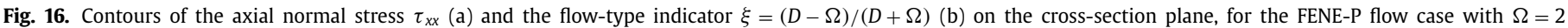
and $E=5$.

There is thus a reversion of the usual rotating cell pattern generated by Coriolis effects. While the standard stress method (method 1) needs 3 hours and 30 minutes of CPU time to predict accurately this flow evolution, the new reduced-stress method needs just about 30 minutes to predict it with the same degree of accuracy.

It was also shown that the reformulation of the constitutive equations associated with the reduced stress method is only minor (compare Eqs. (5) and (12), for $\boldsymbol{\tau}$, and Eqs. (6) and (11), for $f(\operatorname{tr} \tau)$ ) and therefore it should be a simple matter to adapt existing codes (including open source codes, such as that considered in the recent paper [43]) to comply with the present method, when accurate time-dependent calculations are sought. And finally, although we have only considered the original FENE-P equation, the gains in numerical efficiency should extend to more complex equations derived from the microstructural FENE dumbbell model, such as the FENE-L and FENE-LS [44], if these are expressed in terms of a stress tensor equation (instead of being solved for the conformation tensor).

Finally, although the function $f\left(\tau_{k k}\right)$ employed in the definition of the reduced stress is often called extensibility function (as we do here), it is important to realize that it may attain large values even in shear flows, in which the trace of the stress is large due to normal stresses induced by the proximity to a wall. In the two first examples used here as test cases to demonstrate the capabilities of the method, the flow is a pure shear flow. However, the third example is a mixed shear/extensional flow, with a strong, almost pure extensional zone along the middle horizontal line of the cross-section $(z=0)$. This aspect is corroborated in Fig. 16 for the base case (FENE-P on the $101 \times 101$ mesh, with $E=5, L^{2}=100, \beta=0.1$ and rotation speed $\Omega=2$ ) at time $t=20$, showing on the left the deformation of the axial normal stress field $\left(\tau_{x x}\right)$ brought about by the presence of the secondary flow, and on the right contours of a flow indicator function $\xi$ [45], calculated on the cross-section $(\xi=(D-\Omega) /(D+\Omega)$, where $D=$ $\left(\frac{1}{2} \mathbf{D}: \mathbf{D}\right)^{1 / 2}$ and $\Omega=\left(\frac{1}{2} \boldsymbol{\Omega}: \boldsymbol{\Omega}\right)^{1 / 2}$, with the deformation rate tensor $\mathbf{D}=\frac{1}{2}\left(\nabla \mathbf{u}+\nabla \mathbf{u}^{T}\right)$ and vorticity tensor $\left.\boldsymbol{\Omega}=\frac{1}{2}\left(\nabla \mathbf{u}-\nabla \mathbf{u}^{T}\right)\right)$. This flow-type indicator takes values of $-1,0$ and +1 for, respectively, pure rotational, pure shear and pure extensional flows. Fig. 16b shows that in a wide zone adjacent to the lateral walls the flow is shear dominated, but in the central horizontal zone there is a clear sign of a strong extensional flow. Thus, the conclusions about the merits of the reduced stress method are based on tests involving both shear and extensional flows.

\section{Acknowledgments}

The author wishes to thank Universidade da Beira Interior, Portugal, for the sabbatical leave during which this work has been carried out. This work was funded in part by Compete 2020 with national funds from FCT through project UID/EMS/00151/2013.

\section{Appendix: Approximate convergence analysis of the two methods}

The simpler equations valid for the homogeneous start-up flow of the FENE-CR model are sufficient for this analysis since they also reflect the much better behaviour of method 2 over method 1 , which is what we intend to demonstrate in this Appendix. In dimensional terms the stress equations, with the shear rate $\dot{\gamma}=$ $d u / d y$ taken as constant, are:

$\tau_{x y}+\lambda \frac{\partial}{\partial t}\left(\frac{\tau_{x y}}{f}\right)=\eta_{p} \dot{\gamma}$

$\tau_{x x}+\lambda \frac{\partial}{\partial t}\left(\frac{\tau_{x x}}{f}\right)=2 \lambda \frac{\tau_{x y}}{f} \dot{\gamma}$

and when expressed non-dimensionally, by scaling time with the relaxation time, stresses with the ratio $\eta_{p} / \lambda$, and defining a (constant) Weissenberg number as $W i=\lambda \dot{\gamma}$, they become:

$\tau_{x y}+\frac{\partial}{\partial t}\left(\frac{\tau_{x y}}{f}\right)=W i$

$\tau_{x x}+\lambda \frac{\partial}{\partial t}\left(\frac{\tau_{x x}}{f}\right)=2 W i \frac{1}{f} \tau_{x y}$ 
The extensional function, for a sufficiently large $L^{2}$ may be approximated by

$f=\left(L^{2}+\tau_{x x}\right) /\left(L^{2}-3\right) \approx 1+\varepsilon \tau_{x x}$

where $\varepsilon=1 / L^{2}$ is a small parameter (order $\varepsilon \sim 10^{-2}$ ). With method 1 , when the time derivatives are approximated with the Euler scheme, these equations become:

$\tau_{x y}^{n+1}+\frac{1}{\Delta t}\left(\frac{\tau_{x y}^{n+1}}{f^{n+1}}-\frac{\tau_{x y}^{n}}{f^{n}}\right)=W i$

$\tau_{x x}^{n+1}+\frac{1}{\Delta t}\left(\frac{\tau_{x x}^{n+1}}{f^{n+1}}-\frac{\tau_{x x}^{n}}{f^{n}}\right)=2 W i \frac{1}{f^{n+1}} \tau_{x y}^{n+1}$

and the corresponding iteration loop is, dropping the $n+1$ index for new time level:

$\tau_{x y}=\frac{W i+\tau_{x y}^{n} / f^{n} / \Delta t}{1+1 / \Delta t /\left(1+\varepsilon \tau_{x x}\right)}$

$\tau_{x x}=\frac{2 W i \tau_{x y} / f+\tau_{x x}^{n} / \Delta t / f^{n}}{1+1 / \Delta t /\left(1+\varepsilon \tau_{x x}\right)}$

which should be solved by successive substitution. To simplify the notation, we call $X=\tau_{x x}, Y=\tau_{x y}$ and $A=1 / \Delta t$ (a large number, of order $A \sim 10^{2}$ ). We consider the equation for $\tau_{x x}$, in which we assume the numerator of the RHS to be approximately constant $C=2 W i Y / f+A X^{n} / f^{n}$ so that the successive approximations for $\tau_{x x}$, viewed as a fixed-point problem, may be written as:

$X \cong \frac{C}{A}(1+\varepsilon X) \Leftrightarrow X=F(X)\left(\right.$ or $X_{k+1}=F\left(X_{k}\right) ; k=$ iteration number $)$

The condition for convergence is $|\partial F / \partial X|<1$ [46, Section 10.10, p. 569] and the rate of convergence increases as the derivative gets smaller than 1 . For the above expression we have

$\frac{\partial F}{\partial X}=\frac{C}{A} \varepsilon$

or, going back to the original notation,

$\frac{\partial F}{\partial X} \cong \frac{\tau_{x x}^{n}}{f^{n}} \frac{1}{L^{2}}$

We see that is slightly smaller than unity since $f^{n}$ is greater than 1 and $\tau_{x x}^{n}$ at most becomes closer to $L^{2}$ from below. Therefore, we conclude that method 1 converges but the convergence is slow.

With method 2 the non-dimensional equations are:

$f \tau^{\prime}{ }_{x y}+\frac{\partial}{\partial t}\left(\tau_{x y}^{\prime}\right)=W i$

$f \tau_{x x}^{\prime}+\lambda \frac{\partial}{\partial t}\left(\tau^{\prime}{ }_{x x}\right)=2 W i \tau_{x y}^{\prime}$

with $f=\frac{L^{2}}{\left(L^{2}-3-(1 / a) \tau^{\prime}{ }_{x x}\right)} \cong \frac{1}{\left(1-\left(1 / L^{2}\right) \tau^{\prime}{ }_{x x}\right)}$

In discretized form, using again the Euler scheme and the same notation and approximations as before:

$(f+A) Y=W i+A Y^{n}$

$(f+A) X=2 W i Y+A X^{n} \approx C$

with $f=\frac{1}{1-\varepsilon X}$.

The iteration equation for $X$ becomes:

$X=\frac{C}{A+\left(\frac{1}{1-\varepsilon X}\right)} \cong \frac{C}{A+1+\varepsilon X} \equiv F(X)$ and the derivative of the fixed-point iteration function is

$$
\left.\left|\frac{\partial F}{\partial X}\right|=\frac{C \varepsilon}{(A+1+\varepsilon X)^{2}} \text { or }\left|\frac{\partial F}{\partial X}\right| \cong \frac{C}{A^{2}} \varepsilon \quad \text { (since } \varepsilon X<1 \text { but } \varepsilon X \approx 1\right)
$$

or, using $C \cong A X^{n}$,

$$
\left|\frac{\partial F}{\partial X}\right| \cong \frac{X^{n} \varepsilon}{A}=\Delta t\left(\tau_{x x}^{\prime n} / L^{2}\right) \approx O\left(10^{-1}\right) \operatorname{to} O\left(10^{-2}\right) \ll 1
$$

Therefore, this simplified analysis shows that the convergence of method 2 is much faster than method 1 , since for a fixedpoint scheme the iteration error decays as $e_{k+1}=|\partial F / \partial X| e_{k}$ or $e_{k+1}=|\partial F / \partial X|^{k} e_{1}$ [46], where $k$ is the iteration counter.

\section{References}

[1] R.B. Bird, P.J. Dotson, N.L. Johnson, Polymer solution rheology based on a finitely extensible bead-spring chain model, J. Non-Newton. Fluid Mech. 7 (1980) 213-235.

[2] R.B. Bird, C.F. Curtiss, R.C. Armstrong, O. Hassager, Dynamics of polymeric liquids, Kinetic Theory, Vol. 2, second ed., John Wiley \& Sons, New York, 1987.

[3] D.M. Chilcott, J.M. Rallison, Creeping flow of dilute polymer solutions past cylinders and spheres, J. Non-Newton. Fluid Mech. 29 (1988) 381-432.

[4] J.G. Oldroyd, On the formulation of rheological equations of state, Proc. R. Soc. Lond. A 200 (1950) 523-541.

[5] P.J. Oliveira, Alternative derivation of differential constitutive equations of the Oldroyd-B type, J. Non-Newton. Fluid Mech. 160 (2009) 40-46.

[6] N. Phan-Thien, R.I. Tanner, A new constitutive equation derived from network theory, J. Non-Newton. Fluid Mech. 2 (1977) 353-365.

[7] P.J. Coates, R.C. Armstrong, R.A. Brown, Calculation of steady-state viscoelastic flow through axisymmetric contractions with the EEME formulation, J. Non-Newton. Fluid Mech. 42 (1992) 141-188.

[8] M.A. Alves, P.J. Oliveira, F.T. Pinho, Benchmark solutions for the flow of Oldroyd-B and PTT fluids in planar contractions, J. Non-Newton. Fluid Mech. 110 (2003) 45-75.

[9] P.J. Oliveira, F.T. Pinho, G.A. Pinto, Numerical simulation of non-linear elastic flows with a general collocated finite-volume method, J. Non-Newton. Fluid Mech. 79 (1998) 1-43.

[10] D.G. Baird, M. Renardy, Report on the VIIth international workshop on numerical methods in non-Newtonian flow, J. Non-Newton. Fluid Mech. 43 (1992) 383-385.

[11] R.A. Brown, G.H. McKinley, Report on the VIIIth international workshop on numerical methods in viscoelastic flows, J. Non-Newton. Fluid Mech. 52 (1994) 407-413.

[12] B. Caswell, Report on the IXth international workshop on numerical methods in non-Newtonian flows, J. Non-Newton. Fluid Mech. 62 (1996) 99-110.

[13] R.G. Owens, T.N. Phillips, Computational Rheology, Imperial College Press/World Scientific, London, 2002.

[14] S.C. Xue, R.I. Tanner, N. Phan-Thien, Numerical modelling of transient viscoelastic flows, J. Non-Newton. Fluid Mech. 123 (2004) 33-58.

[15] K. Walters, M.F. Webster, The distinctive CFD challenges of computational rheology, Int. J. Num. Meth. Fluids 43 (2003) 577-596.

[16] M.F. Webster, H.R. Tammadon-Jahromi, M. Aboubacar, Transient viscoelastic flows in planar contractions, J. Non-Newton. Fluid Mech. 118 (2004) 83-101.

[17] L. Xi, M.D. Graham, A mechanism for oscillatory instability in viscoelastic cross-slot flow, J. Fluid Mech. 622 (2009) 145-165.

[18] P.J. Oliveira, Method for time-dependent simulations of viscoelastic flows: vortex shedding behind cylinder, J. Non-Newton. Fluid Mech. 101 (2001) 113-137.

[19] N. Fietier, M.O. Deville, Time-dependent algorithms for the simulation of viscoelastic flows with spectral element methods: applications and stability, J. Comput. Phys. 186 (2003) 93-121.

[20] R.G.M Van Os, T.N. Phillips, Efficient and stable spectral element methods for predicting the flow of an XPP fluid past a cylinder, J. Non-Newton. Fluid Mech. 129 (2005) 143-162

[21] R.C. King, M.R. Apelian, R.C. Armstrong, R.A. Brown, Numerically stable finite element techniques for viscoelastic calculations in smooth and singular geometries, J. Non-Newton. Fluid Mech. 29 (1988) 147-216 EEME.

[22] D. Rajagopalan, R.C. Armstrong, R.A. Brown, Finite element methods for calculation of steady, viscoelastic flow using constitutive equations with a Newtonian viscosity, J. Non-Newton. Fluid Mech. 36 (1990) 159-192 EVSS.

[23] J. Sun, N. Phan-Thien, R.I. Tanner, An adaptive viscoelastic stress splitting scheme and its applications: AVSS/SI and AVSS/SUPG, J. Non-Newton. Fluid Mech. 65 (1996) 75-91 AVSS.

[24] H.M. Park, J.Y. Lim, A new numerical algorithm for viscoelastic fluid flows: the grid-by-grid inversion method, J. Non-Newton. Fluid Mech. 165 (2010) $238-246$.

[25] R. Fattal, R. Kupferman, Constitutive laws of the matrix-logarithm of the conformation tensor, J. Non-Newton. Fluid Mech. 123 (2004) 281-285.

[26] R. Fattal, R. Kupferman, Time-dependent simulation of viscoelastic flows at high Weissenberg number using the log-conformation representation, J. Non-Newton. Fluid Mech. 126 (2005) 23-37. 
[27] M.F. Tomé, A. Castelo, A.M. Afonso, M.A. Alves, F.T. Pinho, Application of the log-conformation tensor to three-dimensional time-dependent free surface flows, J. Non-Newton. Fluid Mech. 175/176 (2012) 44-54

[28] G. D’Avino, M.A. Hulsen, Decoupled second-order transient schemes for the flow of viscoelastic fluids without a viscous solvent contribution, J. Non-Newton. Fluid Mech. 165 (2010) 1602-1612.

[29] G. D’Avino, M.A. Hulsen, P.L. Maffettone, Decoupled transient schemes for viscoelastic fluid flow with inertia, Comput. Fluids 66 (2012) 183-193.

[30] E. Castillo, R. Codina, First, second and third order fractional step methods for the three-field viscoelastic flow problem, J. Comput. Phys. 296 (2015) 113-137.

[31] A.S.R. Duarte, A.I.P. Miranda, P.J. Oliveira, Numerical and analytical modeling of unsteady viscoelastic flows: the start-up and pulsating test case problems, J. Non-Newton. Fluid Mech. 154 (2008) 153-169.

[32] N.D. Waters, M.J. King, Unsteady flow of an elastico-viscous liquid, Rheol. Acta 9 (1970) 345-355.

[33] F.M. White, Viscous Fluid Flow, second ed., McGraw-Hill, 1991.

[34] R.B. Bird, Kinetic theory of polymeric liquids, Acc. Chem. Res 18 (1985) 364-371.

[35] R.D. Richtmyer, K.W. Morton, Difference Methods for Initial-Value Problems, second ed., InterScience Publisher, New York, 1967.

[36] M.A. Alves, P.J. Oliveira, F.T. Pinho, A convergent and universally bounded interpolation scheme for the treatment of advection, Int. J. Num. Meth. Fluids 41 (2003) 47-75.
[37] T.J. Bromwich, An application of Heaviside's methods of viscous fluid motion, J. Lond. Math. Soc. 5 (1930) 10-13.

[38] IJ. Keshtiban, B. Puangkird, H.R. Tammadon-Jahromi, M.F Webster, Generalised approach for transient computation of start-up pressure-driven viscoelastic flow, J. Non-Newton. Fluid Mech. 151 (2008) 2-20.

[39] D.O. Cruz, F.T. Pinho, P.J. Oliveira, Analytical solution for fully developed laminar flow of some viscoelastic liquids with a newtonian solvent contribution, J. Non-Newton. Fluid Mech. 132 (2005) 28-35.

[40] A.I.P. Miranda, P.J. Oliveira, Start-up times in viscoelastic channel and pipe flows, Korea Aust. Rheol. J. 22 (2010) 65-73.

[41] P.J. Oliveira, Asymmetric flows of viscoelastic fluids in symmetric planar expansion geometries, J. Non-Newton. Fluid Mech. 114 (2003) 33-63.

[42] P. Pakdel, S.H. Spiegelberg, G.H. McKinley, Cavity flows of elastic liquids: two-dimensional flows, Phys. Fluids 9 (1997) 3123.

[43] F. Pimenta, M.A. Alves, Stabilization of an open-source finite-volume solver for viscoelastic fluid flows, J. Non-Newton. Fluid Mech. 239 (2017) 85-104.

[44] G. Lielens, R. Keunings, V. Legat, The FENE-L and FENE-LS closure approximations to the kinetic theory of finitely extensible dumbbells, J. Non-Newton. Fluid Mech. 87 (1999) 179-196.

[45] J.S. Lee, R. Dylla-Spears, N-P. Teclemariam, S.J. Muller, Microfluidic four-roll mill for all flow types, Appl. Phys. Lett. 90 (2007) 074103.

[46] F.B. Hildebrand, Introduction to Numerical Analysis, second ed., McGraw-Hill, New York, 1974. 